\title{
Nickel-Catalyzed Cyclization of 2-Iodoanilines with Aroylalkynes: An Efficient Route for Quinoline Derivatives
}

\author{
Korivi Rajendra Prasad, Chien-Hong Cheng* \\ Department of Chemistry, National Tsing Hua University,Hsinchu, 30013, Taiwan \\ chcheng@mx.nthu.edu.tw
}

Supporting Information

\section{Table of Contents}

$\begin{array}{ll}\text { 1. Preparation of starting materials } & \text { S2 }\end{array}$

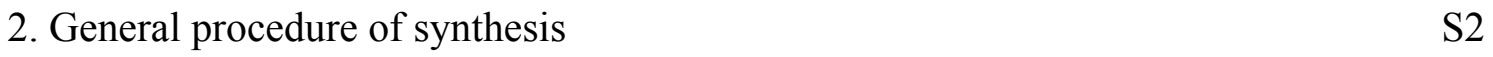

3. Spectral data of the compounds $\quad$ S3-S6

4. NOE data for the selected compounds $\quad$ S7

5. X-ray structure, crystal data and structure refinement $\quad$ S8

6. ${ }^{1} \mathrm{H}$ and ${ }^{13} \mathrm{C}$ NMR spectra of selected compounds $\quad$ S10-S37 


\section{Preparation of Starting Materials:}

The starting materials for the present catalytic reaction are prepared using reported

procedures. The $o$-iodoanilines ${ }^{1,2} \mathbf{1 b}, \mathbf{1 c}, \mathbf{1 d}$ and $\mathbf{1 e}$ are prepared from iodination of corresponding aniline derivatives using $\mathrm{I}_{2}$ and base in water. The other starting material benzoylalkynes are obtained in excellent yields $(\sim 85 \%)$ from the lithiation of alkyne and fallowed by the addition of benzoylchloride in THF at $-78{ }^{\circ} \mathrm{C}$ or the other procedures ${ }^{3}$ can be used. Both the crude mixtures there after are subjected to chromatography to get pure products.

\section{General Procedure for the Preparation of Quinolines:}

A round bottom side-arm flask $(25 \mathrm{~mL})$ containing $\mathrm{NiBr}_{2}(\mathrm{dppe})(3.1 \mathrm{mg}, 0.0050 \mathrm{mmol})$, 2-iodoaniline $1(0.10 \mathrm{mmol})$, alkyne $2(0.20 \mathrm{mmol})$ and zinc powder $(13 \mathrm{mg}, 0.20 \mathrm{mmol})$ was subjected to the Schlenk-line procedures of evacuation and purging of nitrogen for 3 cycles. Freshly distilled acetonitrile $(3.0 \mathrm{~mL})$ was added to the reaction system and the reaction mixture was stirred at $80{ }^{\circ} \mathrm{C}$ for $12 \mathrm{~h}$. Then the reaction mixture was cooled to room temperature and diluted with dichloromethane. The mixture thus obtained was filtered through a silica gel pad and washed thoroughly with dichloromethane. The filtrate was concentrated in a rotary evaporator and the residue was separated on a silica gel column using a mixture of hexanes and ethyl acetate as eluent to afford the desired pure product 4. Compounds $\mathbf{4 a - 1}$ were synthesized according to this procedure and the spectral data of these compounds are as follows. The spectral data of products $\mathbf{4 a},{ }^{4} \mathbf{4 d},{ }^{5}$ $\mathbf{4 e},{ }^{6} \mathbf{4 f}, \mathbf{4 m}^{8}$ and $\mathbf{4} \mathbf{n}^{9}$ were reported in the literature. 
2,4-Diphenylquinoline (4a): white solid; $\mathrm{mp}=102{ }^{\circ} \mathrm{C} ; \mathrm{R}_{\mathrm{f}}=0.57(10 \%$ ethyl acetate in hexanes); ${ }^{1} \mathrm{H}$ NMR $\delta$ 7.44-7.48 (m, 2H), 7.50-7.57 (m, 7H), $7.72(\mathrm{t}, J=7.5 \mathrm{~Hz}$, 1H), $7.81(\mathrm{~s}, 1 \mathrm{H}), 7.90(\mathrm{~d}, J=3.0 \mathrm{~Hz}, 1 \mathrm{H}), 8.18(\mathrm{~d}, J=8.0 \mathrm{~Hz}, 2 \mathrm{H})$ and $8.24(\mathrm{~d}, J=9.0$ $\mathrm{Hz}, 1 \mathrm{H}) ;{ }^{13} \mathrm{C}$ NMR $\delta 119.4,125.6,125.8,126.3,127.6,128.4,128.6,128.8,129.3,129.5$, 129.6, 130.1, 138.4, 139.7, 148.8, 149.2 and 156.9; HRMS (EI+) 281.1195 (cal for $\left.\mathrm{C}_{21} \mathrm{H}_{15} \mathrm{~N} 281.1204\right) ;$ IR $1357,1489,1590$ and $3053 \mathrm{~cm}^{-1}$.

4-Butyl-2-phenylquinoline (4b): viscus liquid; $\mathrm{R}_{\mathrm{f}}=0.60$ (10\% ethyl acetate in hexanes); ${ }^{1} \mathrm{H}$ NMR $\delta 0.98(\mathrm{t}, J=7.0 \mathrm{~Hz}, 3 \mathrm{H}), 1.46-1.50(\mathrm{~m}, 2 \mathrm{H}), 1.75-1.80(\mathrm{~m}, 2 \mathrm{H}), 3.12$ (t, $J=7.8 \mathrm{~Hz}, 2 \mathrm{H}), 7.44(\mathrm{t}, J=6.8 \mathrm{~Hz}, 1 \mathrm{H}), 7.49-7.53(\mathrm{~m}, 3 \mathrm{H}), 7.67-7.70(\mathrm{~m}, 2 \mathrm{H}), 8.03$ $(\mathrm{d}, J=8.5 \mathrm{~Hz}, 1 \mathrm{H}), 8.13(\mathrm{~d}, J=8.5 \mathrm{~Hz}, 2 \mathrm{H})$ and $8.18(\mathrm{~d}, J=8.0 \mathrm{~Hz}, 1 \mathrm{H}) ;{ }^{13} \mathrm{C}$ NMR $\delta$ $13.9,22.8,32.3,32.3,118.8,123.4,126.0,126.6,127.6,128.5,128.8,129.2,130.4$, 139.9, 148.4, 149.4 and 157.1; HRMS (EI $\left.{ }^{+}\right) 261.1512$ (cal for $\mathrm{C}_{19} \mathrm{H}_{19} \mathrm{~N}$ 261.1517); IR $1353,1447,1551,1597$ and $2957 \mathrm{~cm}^{-1}$.

2-(Naphthalen-2-yl)-4-phenylquinoline (4c): yellow solid; $m p=110{ }^{\circ} \mathrm{C} ; \mathrm{R}_{\mathrm{f}}=0.58$ (10\% ethyl acetate in hexanes); ${ }^{1} \mathrm{H}$ NMR $\delta 7.49(\mathrm{dt}, J=5.9$ and $0.9 \mathrm{~Hz}, 1 \mathrm{H}), 7.51-7.53$ (m, 3H), 7.55-7.60 (m, 4H), $7.75(\mathrm{dt}, J=6.4$ and $1.1 \mathrm{~Hz}, 1 \mathrm{H}), 7.86-7.90(\mathrm{~m}, 1 \mathrm{H}), 7.92(\mathrm{~d}$, $J=7.0 \mathrm{~Hz}, 1 \mathrm{H}), 7.96-8.00(\mathrm{~m}, 3 \mathrm{H}), 8.30(\mathrm{~d}, J=7.0 \mathrm{~Hz}, 1 \mathrm{H}), 8.39(\mathrm{dd}, J=7.1$ and 1.4 $\mathrm{Hz}, 1 \mathrm{H})$ and $8.64(\mathrm{~s}, 1 \mathrm{H}) ;{ }^{13} \mathrm{C}$ NMR $\delta 119.5,125.1,125.7,125.8,126.3,126.4,126.7$, $127.2,127.7,128.5,128.6,128.6,128.8,129.6,129.7,130.0,133.5,133.9,136.8,138.4$, 148.8, 149.4 and 156.6; HRMS (EI $\left.{ }^{+}\right) 331.1346$ (cal for $\mathrm{C}_{25} \mathrm{H}_{17} \mathrm{~N}$ 331.1361); IR 1361, $1488,1548,1592$ and $2926 \mathrm{~cm}^{-1}$.

6-Methyl-2,4-diphenylquinoline (4d): white solid; $\mathrm{mp}=111{ }^{\circ} \mathrm{C} ; \mathrm{R}_{\mathrm{f}}=0.53(10 \%$ ethyl acetate in hexanes); ${ }^{1} \mathrm{H}$ NMR $\delta 2.46(\mathrm{~s}, 3 \mathrm{H}), 7.44(\mathrm{t}, J=7.0 \mathrm{~Hz}, 1 \mathrm{H}), 7.51(\mathrm{t}, J=7.3$ 
$\mathrm{Hz}, 3 \mathrm{H}), 7.54-7.57(\mathrm{~m}, 5 \mathrm{H}), 7.64(\mathrm{~s}, 1 \mathrm{H}), 7.77(\mathrm{~s}, 1 \mathrm{H})$ and $8.13-8.17(\mathrm{~m}, 3 \mathrm{H}) ;{ }^{13} \mathrm{C} \mathrm{NMR} \delta$ $21.8,119.4,124.4,125.7,127.5,128.3,128.6,128.8,129.2,129.5,129.8,131.8,136.3$, 138.6, 139.7, 147.3, 148.5 and 156.0; HRMS (EI $\left.{ }^{+}\right) 295.1369$ (cal for $\mathrm{C}_{22} \mathrm{H}_{17} \mathrm{~N} 295.1361$ ); IR $1358,1489,1545,1588$ and $2912 \mathrm{~cm}^{-1}$.

4-Butyl-6-methyl-2-phenylquinoline (4e): white solid; $\mathrm{mp}=86{ }^{\circ} \mathrm{C} ; \mathrm{R}_{\mathrm{f}}=0.48$ (10\% ethyl acetate in hexanes); ${ }^{1} \mathrm{H}$ NMR $\delta 0.99(\mathrm{t}, J=7.5 \mathrm{~Hz}, 3 \mathrm{H}), 1.46-1.51(\mathrm{~m}, 2 \mathrm{H})$, $1.75-1.81(\mathrm{~m}, 2 \mathrm{H}), 2.56(\mathrm{~s}, 3 \mathrm{H}), 3.08(\mathrm{t}, J=7.8 \mathrm{~Hz}, 2 \mathrm{H}), 7.42(\mathrm{t}, J=7.3 \mathrm{~Hz}, 1 \mathrm{H}), 7.48-$ $7.53(\mathrm{~m}, 3 \mathrm{H}), 7.65(\mathrm{~s}, 1 \mathrm{H}), 7.76(\mathrm{~s}, 1 \mathrm{H}), 8.05(\mathrm{~d}, J=8.5 \mathrm{~Hz}, 1 \mathrm{H})$ and $8.11(\mathrm{~d}, J=8.0 \mathrm{~Hz}$, $2 \mathrm{H}) ;{ }^{13} \mathrm{C}$ NMR $\delta 14.0,22.0,22.8,32.2(2 \mathrm{C}), 118.7,122.4,126.5,127.5,128.7,128.9$, 130.2, 131.4, 135.7, 140.1, 147.0, 148.5 and 156.2; $\mathrm{HRMS}\left(\mathrm{EI}^{+}\right) 275.1650$ (cal for $\mathrm{C}_{20} \mathrm{H}_{21} \mathrm{~N}$ 275.1674); IR 1354, 1447, 1553, 1596 and $2924 \mathrm{~cm}^{-1}$.

6-Chloro-2,4-diphenylquinoline (4f): yellow solid; $m p=121{ }^{\circ} \mathrm{C} ; \mathrm{R}_{\mathrm{f}}=0.60(10 \%$ ethyl acetate in hexanes); ${ }^{1} \mathrm{H}$ NMR $\delta 7.47(\mathrm{t}, J=6.8 \mathrm{~Hz}, 1 \mathrm{H}), 7.51-7.53(\mathrm{~m}, 5 \mathrm{H}), 7.55-$ $7.57(\mathrm{~m}, 2 \mathrm{H}), 7.66(\mathrm{dd}, J=7.5$ and $1.9 \mathrm{~Hz}, 1 \mathrm{H}), 7.83(\mathrm{~s}, 1 \mathrm{H}), 7.86(\mathrm{~d}, J=1.9 \mathrm{~Hz}, 1 \mathrm{H})$ and 8.17-8.20 (m, 3H); ${ }^{13} \mathrm{C}$ NMR $\delta 120.1,124.5,126.5,127.6,128.7,128.8,128.9,129.4$, 129.7, 130.5, 131.6, 132.3, 137.7, 139.0, 147.0, 148.6 and 157.0; HRMS (EI $\left.{ }^{+}\right) 315.0815$ (cal for $\mathrm{C}_{21} \mathrm{H}_{14} \mathrm{ClN} 315.0815$ ); IR 1153, 1359, 1484, 1589 and $2924 \mathrm{~cm}^{-1}$.

4-Butyl-6-chloro-2-phenylquinoline (4g): yellow solid; $\mathrm{mp}=87{ }^{\circ} \mathrm{C} ; \mathrm{R}_{\mathrm{f}}=0.58$ (10\% ethyl acetate in hexanes); ${ }^{1} \mathrm{H}$ NMR $\delta 0.99$ (t, $\left.J=7.3 \mathrm{~Hz}, 3 \mathrm{H}\right), 1.45-1.50(\mathrm{~m}, 2 \mathrm{H})$, $1.74-1.80(\mathrm{~m}, 2 \mathrm{H}), 3.06(\mathrm{t}, J=7.8 \mathrm{~Hz}, 2 \mathrm{H}), 7.45(\mathrm{dt}, J=8.0$ and $2.0 \mathrm{~Hz}, 1 \mathrm{H}), 7.51(\mathrm{t}, J=$ $7.0 \mathrm{~Hz}, 2 \mathrm{H}), 7.62(\mathrm{dd}, J=8.8$ and $2.0 \mathrm{~Hz}, 1 \mathrm{H}), 7.71(\mathrm{~s}, 1 \mathrm{H}), 7.97(\mathrm{~d}, J=2.0 \mathrm{~Hz}, 1 \mathrm{H})$, 8.09-8.13 (m, 3H); ${ }^{13} \mathrm{C}$ NMR $\delta$ 13.9, 22.8, 32.1, 32.2, 119.4, 122.5, 127.3, 127.6, 128.9, 
129.5, 130.1, 131.8, 131.9, 139.3, 146.7, 148.8 and 157.2; $\mathrm{HRMS}\left(\mathrm{EI}^{+}\right) 295.1142$ (cal for $\left.\mathrm{C}_{19} \mathrm{H}_{18} \mathrm{CIN} 295.1128\right)$; IR 1116, 1166, 1306, 1466, 1598 and $2963 \mathrm{~cm}^{-1}$.

6-(Trifluoromethyl)-2,4-diphenylquinoline (4h): white solid; $\mathrm{mp}=138{ }^{\circ} \mathrm{C} ; \mathrm{R}_{\mathrm{f}}=$ $0.57\left(10 \%\right.$ ethyl acetate in hexanes); ${ }^{1} \mathrm{H}$ NMR $\delta 7.49-7.60(\mathrm{~m}, 8 \mathrm{H}), 7.90(\mathrm{~d}, J=10.0 \mathrm{~Hz}$, $2 \mathrm{H}), 8.21(\mathrm{~d}, J=7.5 \mathrm{~Hz}, 3 \mathrm{H})$ and $8.35(\mathrm{~d}, J=9.0 \mathrm{~Hz}, 1 \mathrm{H}) ;{ }^{13} \mathrm{C}$ NMR $\delta 120.4,123.7$, $123.7,124.9,125.3,127.7,128.9,129.0,129.5,130.0,131.2,137.4,138.8,149.8,150.2$ and 158.8; HRMS (EI $\left.{ }^{+}\right) 349.1064$ (cal for $\mathrm{C}_{22} \mathrm{H}_{14} \mathrm{~F}_{3} \mathrm{~N}$ 349.1078); IR 1117, 1312, 1467, 1592 and $2924 \mathrm{~cm}^{-1}$.

4-Butyl-6-(trifluoromethyl)-2-phenylquinoline (4i): white solid; $m p=92{ }^{\circ} \mathrm{C} ; \mathrm{R}_{\mathrm{f}}=$ $0.65\left(10 \%\right.$ ethyl acetate in hexanes); ${ }^{1} \mathrm{H}$ NMR $\delta 1.00(\mathrm{t}, J=7.2 \mathrm{~Hz}, 3 \mathrm{H}), 1.48-1.52(\mathrm{~m}$, 2H), 1.77-1.82 (m, 2H), $3.14(\mathrm{t}, J=7.8 \mathrm{~Hz}, 2 \mathrm{H}), 7.48(\mathrm{t}, J=7.5 \mathrm{~Hz}, 1 \mathrm{H}), 7.53(\mathrm{t}, J=7.8$ $\mathrm{Hz}, 2 \mathrm{H}), 7.78(\mathrm{~s}, 1 \mathrm{H}), 7.86(\mathrm{dd}, J=8.4 \mathrm{and} 2.0 \mathrm{~Hz}, 1 \mathrm{H}), 8.15-8.17(\mathrm{~m}, 2 \mathrm{H}), 8.26(\mathrm{~d}, J=$ $8.4 \mathrm{~Hz}, 1 \mathrm{H})$ and $8.31(\mathrm{~s}, 1 \mathrm{H}) ;{ }^{13} \mathrm{C}$ NMR $\delta 13.9,22.7,32.0,32.2,119.6,121.4,121.4$, $124.9,125.7,127.7,128.9,129.8,131.5,139.2,149.5,150.3$ and 159.0; HRMS (EI') 329.1375 (cal for $\mathrm{C}_{20} \mathrm{H}_{18} \mathrm{~F}_{3} \mathrm{~N} 329.1391$ ); IR 1116, $1166,1306,1598$ and $2963 \mathrm{~cm}^{-1}$.

6-chloro-4-ethyl-2-methylquinoline $(4 \mathrm{j})$ : white solid; $\mathrm{mp}=74{ }^{\circ} \mathrm{C} ; \mathrm{R}_{\mathrm{f}}=0.58(20 \%$ ethyl acetate in hexanes); ${ }^{1} \mathrm{H}$ NMR $\delta 1.36$ (t, $\left.J=7.3 \mathrm{~Hz}, 3 \mathrm{H}\right), 2.66$ (s, 3H), 3.00 (q, $J=$ $7.5 \mathrm{~Hz}, 2 \mathrm{H}), 7.13(\mathrm{~s}, 1 \mathrm{H}), 7.57(\mathrm{dd}, J=9.0$ and $2.0 \mathrm{~Hz}, 1 \mathrm{H})$ and $7.91-7.94(\mathrm{~m}, 2 \mathrm{H}) ;{ }^{13} \mathrm{C}$ NMR $\delta 13.9,24.8,25.3,121.4,122.3,126.5,129.7,130.9,131.2,146.3,149.0$ and 159.2; HRMS (EI $\left.{ }^{+}\right) 205.0659$ (cal for $\mathrm{C}_{12} \mathrm{H}_{12} \mathrm{ClN}$ 205.0658); IR 1091, 1383, 1603 and $2925 \mathrm{~cm}^{-}$ 1.

2-(Benzo[d][1,3]dioxol-6-yl)-4-(trimethylsilyl)quinoline (4k): white solid; $\mathrm{R}_{\mathrm{f}}=$ 0.47 (10\% ethyl acetate in hexanes); ${ }^{1} \mathrm{H}$ NMR $\delta 0.51(\mathrm{~s}, 9 \mathrm{H}), 6.03(\mathrm{~s}, 2 \mathrm{H}), 6.94(\mathrm{~d}, J=$ 
$8.0 \mathrm{~Hz}, 1 \mathrm{H}), 7.50(\mathrm{dt}, J=7.5$ and $1.0 \mathrm{~Hz}, 1 \mathrm{H}), 7.63(\mathrm{dd}, J=8.3$ and $1.8 \mathrm{~Hz}, 1 \mathrm{H}), 7.66-$ $7.70(\mathrm{~m}, 2 \mathrm{H}), 7.90(\mathrm{~s}, 1 \mathrm{H}), 8.01(\mathrm{~d}, J=8.5 \mathrm{~Hz}, 1 \mathrm{H})$ and $8.15(\mathrm{~d}, J=8.0 \mathrm{~Hz}, 1 \mathrm{H}) ;{ }^{13} \mathrm{C}$ NMR $\delta-0.2,101.3,108.0,108.5,121.8,125.1,125.7,127.6,129.0,130.4,130.6,134.3$, 147.4, 148.3, 148.7, 149.4 and 155.3; HRMS $\left(\mathrm{EI}^{+}\right) 321.1173$ (cal for $\mathrm{C}_{19} \mathrm{H}_{19} \mathrm{NO}_{2} \mathrm{Si}$ 321.1185); IR 1041, 1252, 1445, 1489 and $2922 \mathrm{~cm}^{-1}$.

4-(Trimethylsilyl)-2-phenylquinoline (4I): viscus liquid; $\mathrm{R}_{\mathrm{f}}=0.60$ (10\% ethyl acetate in hexanes); ${ }^{1} \mathrm{H}$ NMR $\delta 0.52(\mathrm{~s}, 9 \mathrm{H}), 7.45-7.47$ (m, 1H), 7.51-7.55 (m, 3H), 7.69$7.73(\mathrm{t}, J=7.8 \mathrm{~Hz}, 1 \mathrm{H}), 7.99(\mathrm{~s}, 1 \mathrm{H}), 8.05(\mathrm{~d}, J=8.5 \mathrm{~Hz}, 1 \mathrm{H}), 8.13(\mathrm{~d}, J=7.0 \mathrm{~Hz}, 2 \mathrm{H})$ and $8.27(\mathrm{~d}, J=8.0 \mathrm{~Hz}, 1 \mathrm{H}) ;{ }^{13} \mathrm{C}$ NMR $\delta-0.2,125.6,126.0,127.6,127.8,128.9,129.2$, 129.4, 130.5, 130.6 and 155.9; HRMS $\left(\mathrm{EI}^{+}\right) 277.1299$ (cal for $\mathrm{C}_{18} \mathrm{H}_{19} \mathrm{NSi} 277.1287$ ).

2-(Benzo[d][1,3]dioxol-6-yl)quinoline (4m): white solid; $\mathrm{mp}=85{ }^{\circ} \mathrm{C} ; \mathrm{R}_{\mathrm{f}}=0.33$ (10\% ethyl acetate in hexanes); ${ }^{1} \mathrm{H}$ NMR $\delta 6.02(\mathrm{~s}, 2 \mathrm{H}), 6.93(\mathrm{~d}, J=8.0 \mathrm{~Hz}, 1 \mathrm{H}), 7.48(\mathrm{t}$, $J=7.0 \mathrm{~Hz}, 1 \mathrm{H}), 7.64(\mathrm{dd}, J=8.0$ and $1.5 \mathrm{~Hz}, 1 \mathrm{H}), 7.69(\mathrm{dt}, J=8.0$ and $1.0 \mathrm{~Hz}, 1 \mathrm{H}), 7.72$ (d, $J=1.5 \mathrm{~Hz}, 1 \mathrm{H}), 7.76-7.79(\mathrm{~m}, 2 \mathrm{H}), 8.11(\mathrm{~d}, J=8.5 \mathrm{~Hz}, 1 \mathrm{H})$ and $8.15(\mathrm{~d}, J=8.5 \mathrm{~Hz}$, $1 \mathrm{H}) ;{ }^{13} \mathrm{C}$ NMR $\delta 101.4,107.9,108.5,118.6,121.8,126.1,127.0,127.4,129.5,129.7$, 134.1, 136.7, 148.2, 148.4, 148.8 and 156.7; HRMS (EI $\left.{ }^{+}\right) 249.0782$ (cal for $\mathrm{C}_{16} \mathrm{H}_{11} \mathrm{NO}_{2}$ 249.0790); IR 1051, 1260, 1497, 1596 and $2923 \mathrm{~cm}^{-1}$.

2-Phenylquinoline (4n): white solid; $\mathrm{mp}=72{ }^{\circ} \mathrm{C} ; \mathrm{R}_{\mathrm{f}}=0.50(10 \%$ ethyl acetate in hexanes); ${ }^{1} \mathrm{H}$ NMR $\delta$ 7.44-7.47 (t, $\left.J=7.5 \mathrm{~Hz}, 1 \mathrm{H}\right), 7.50-7.53$ (m, 3H), 7.70-7.73 (dt, $J=$ 1.5 and $7.3 \mathrm{~Hz}, 1 \mathrm{H}), 7.82(\mathrm{~d}, J=8.0 \mathrm{~Hz}, 1 \mathrm{H}), 7.86(\mathrm{~d}, J=8.5 \mathrm{~Hz}, 1 \mathrm{H}), 8.15(\mathrm{~d}, J=7.5$ $\mathrm{Hz}, 2 \mathrm{H})$ and $8.20(\mathrm{t}, J=9.5 \mathrm{~Hz}, 1 \mathrm{H}) ;{ }^{13} \mathrm{C}$ NMR $\delta 119.0,126.3,127.2,127.4,127.6$, 128.8, 129.3, 129.7, 129.8, 136.8, 139.7, 148.2 and 157.4; $\mathrm{HRMS}\left(\mathrm{EI}^{+}\right) 205.0899$ (cal for $\mathrm{C}_{15} \mathrm{H}_{11} \mathrm{~N}$ 205.0891); IR 1492, 1598, 1728 and $2929 \mathrm{~cm}^{-1}$. 
NOE Experiments. The regiochemistry of most products 4 were established based on the ${ }^{1} \mathrm{H}$ NMR NOE experiments. The results of the NOE experiments are summarized below.
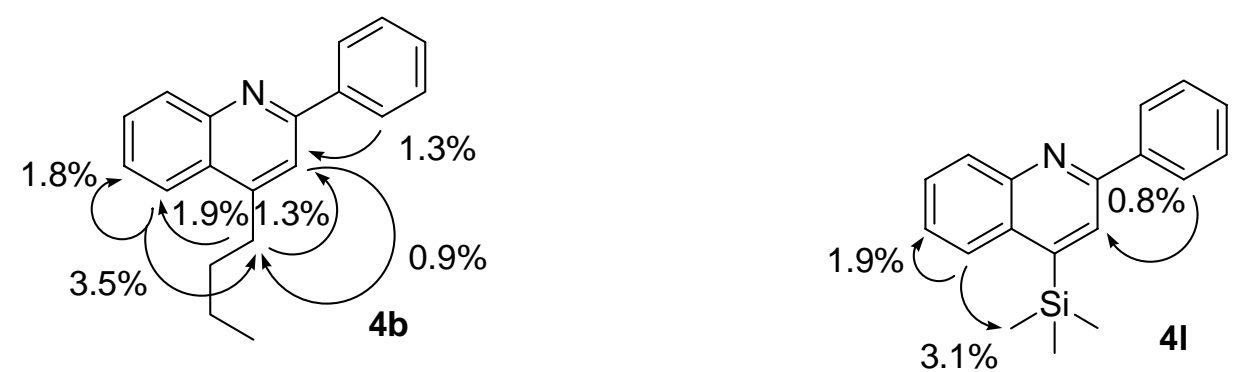
Figure 1, X-ray Structure for 4-Butyl-6-(trifluoromethyl)-2-phenylquinoline (4i).

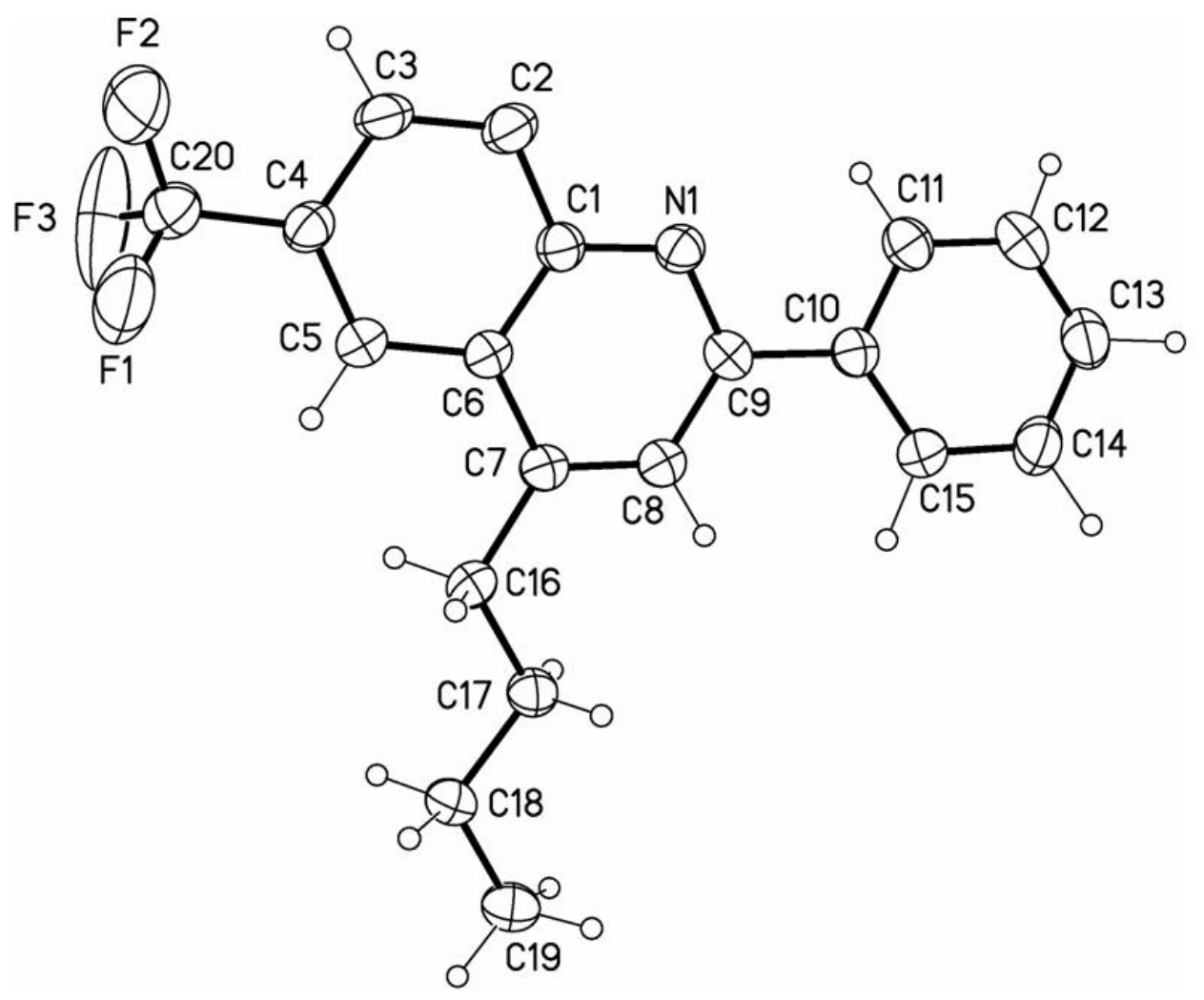

Table 1. Crystal data and structure refinement for sp16m (compound 4i).

Identification code

Empirical formula

Formula weight

Temperature

Wavelength

Crystal system

Space group

Unit cell dimensions

Volume

Z

Density (calculated) sp16m

C20 H18 F3 N

329.35

273(2) K

$0.71073 \AA$

Monoclinic

P2(1)/c

$\begin{array}{ll}\mathrm{a}=10.8344(15) \AA & \alpha=90^{\circ} . \\ \mathrm{b}=18.814(3) \AA & \beta=96.537(3)^{\circ} . \\ \mathrm{c}=8.0715(11) \AA & \gamma=90^{\circ} .\end{array}$

1634.6(4) $\AA^{3}$

4

$1.338 \mathrm{Mg} / \mathrm{m}^{3}$ 


$\begin{array}{ll}\text { Absorption coefficient } & 0.102 \mathrm{~mm}^{-1} \\ \mathrm{~F}(000) & 688 \\ \text { Crystal size } & 0.3 \times 0.1 \times 0.1 \mathrm{~mm}^{3} \\ \text { Theta range for data collection } & 1.89 \text { to } 25.39^{\circ} \\ \text { Index ranges } & -13 \leq \mathrm{h} \leq 12,-22 \leq \mathrm{k} \leq 20,-9 \leq 1 \leq 9 \\ \text { Reflections collected } & 9725 \\ \text { Independent reflections } & 3011[\mathrm{R}(\mathrm{int})=0.0465] \\ \text { Completeness to theta }=25.39^{\circ} & 100.0 \% \\ \text { Refinement method } & \mathrm{Full}-\mathrm{matrix} \text { least-squares on } \mathrm{F}^{2} \\ \text { Data / restraints / parameters } & 3011 / 0 / 218 \\ \text { Goodness-of-fit on F } 2 & 0.928 \\ \text { Final R indices [I }>3 \text { sigma(I)] } & \mathrm{R} 1=0.0634, \mathrm{wR} 2=0.2292 \\ \mathrm{R} \text { indices (all data) } & \mathrm{R} 1=0.1189, \mathrm{wR} 2=0.2196 \\ \text { Extinction coefficient } & 0.025(4) \\ \text { Largest diff. peak and hole } & 0.545 \text { and }-0.410 \text { e. } \AA^{-3}\end{array}$

\section{References}

1. Xiao, W. -J.; Alper, H. J. Org. Chem.1999, 64, 9646.

2. Dains, F. B.; Vaughan, T. H.; Janney, W. M. J. Am. Chem. Soc. 1918, 40, 932.

3. (a) L. Chen, L.; Li, C-J. Org. Lett., 2004, 6, 3151. (b) Lee, P. H.; Lee, S. W.; Seomoon, D. Org. Lett. 2003, 5, 4963.

4. Ahmet, M. T.; Miller, J. R.; Osborne, A. G.; Warmsley, J. F. Acta Crystallographica SectionC, 1995, 51, 2105.

5. Nishio, T.; Omote, Y. J. Chem. Soc. Perkin. Trans 1, 1983, 8, 1773.

6.Yadav, J. S.; Reddy, B. V. S.; Rao, R. S.; Kumar, V. N. Synthesis, 2003, 10, 1610.

7. Kobayashi, K.; Morikawa, O.; Konishi, H. Tetrahedron, 2004, 60, 11639.

8. Echavarren, A. M.; Stille, J. K. J. Am. Chem. Soc, 1987, 109, 5478.

9. Fakhfakh, M. A.; Fournet, A.; Prina, E.; Mouscadet, J-F.; Franck, X.; Hocquemiller, R.; Figadere, B. Bioorg. Med. Chem. 2003, 11, 5013. 


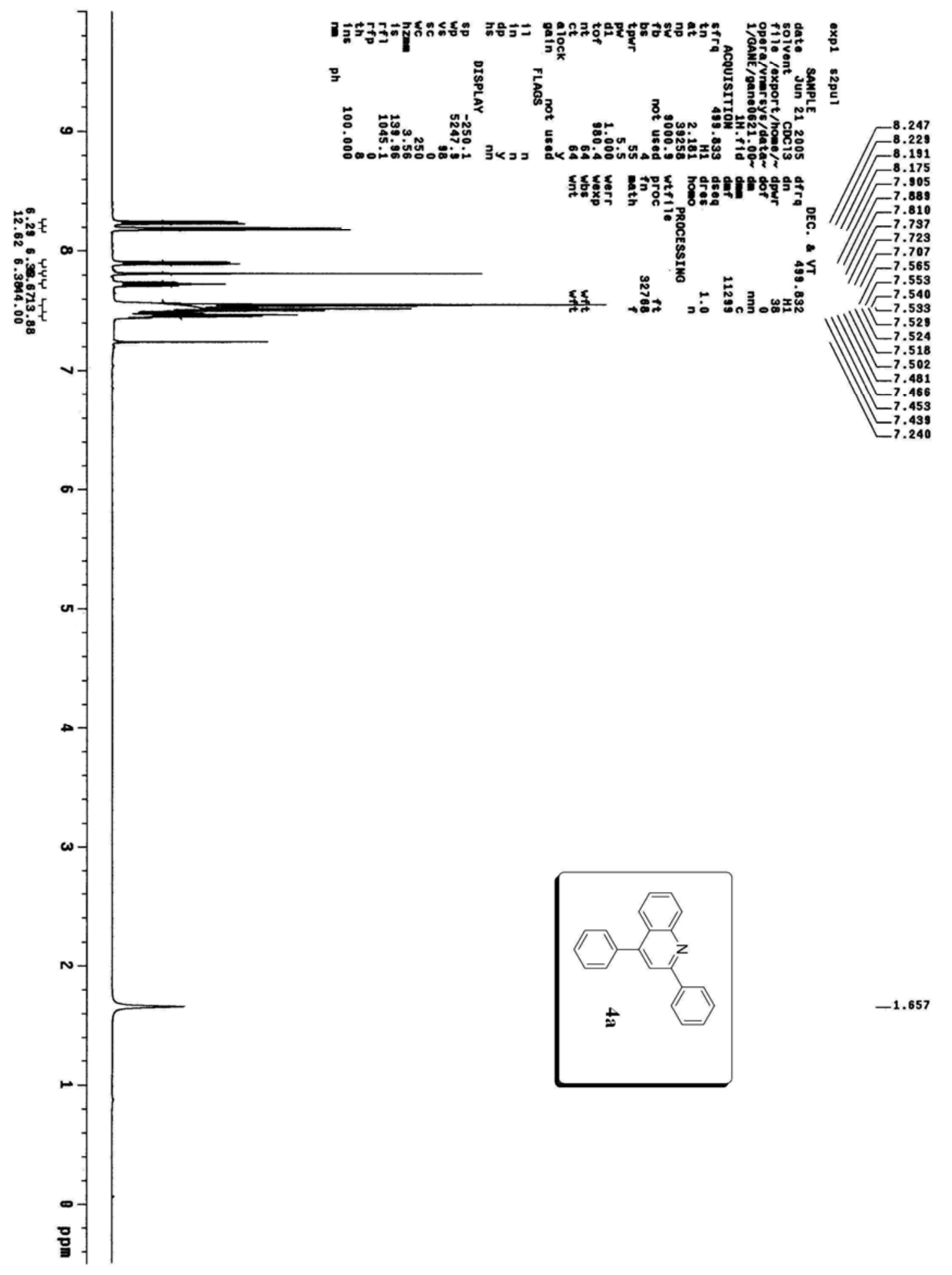




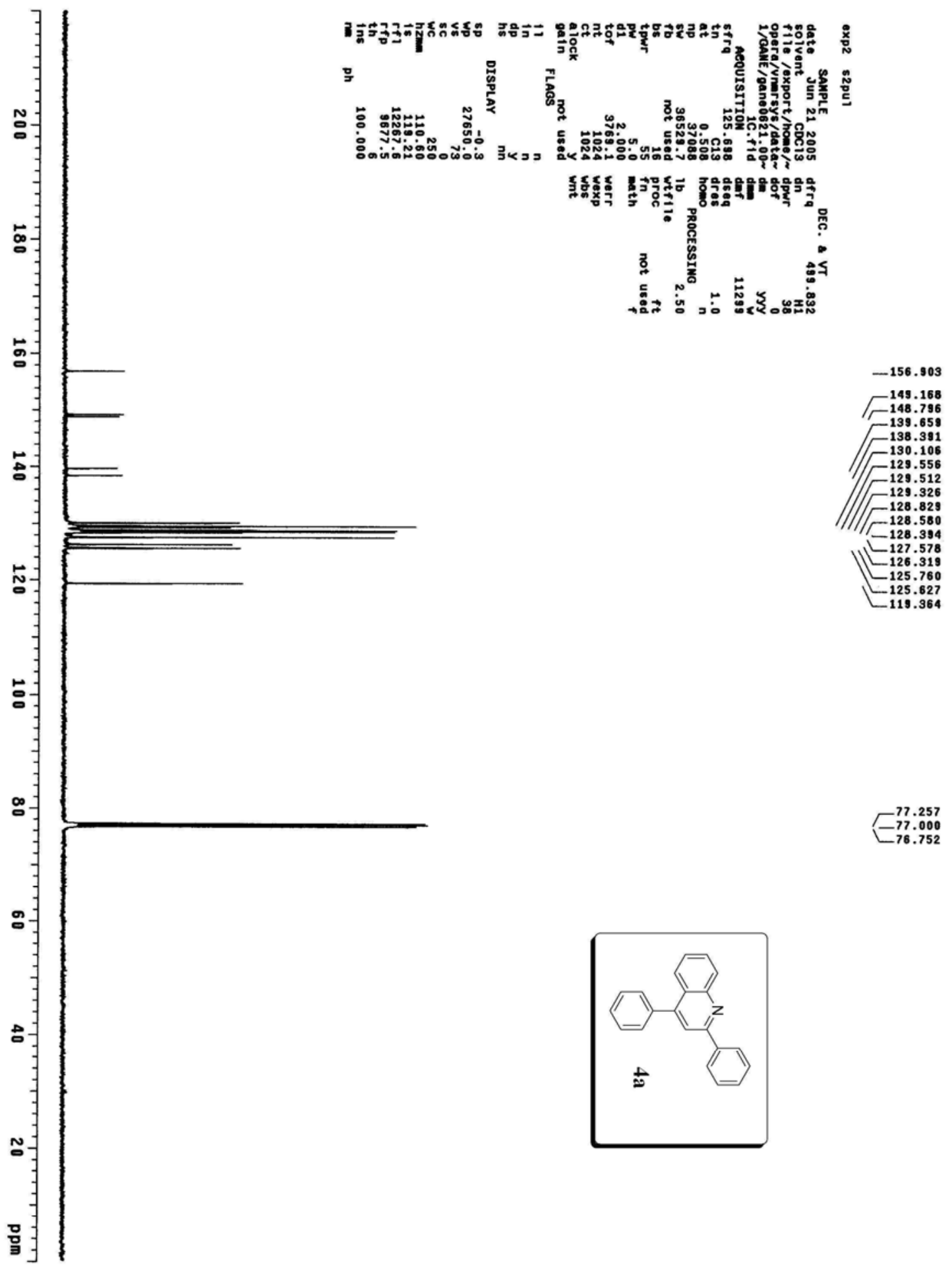




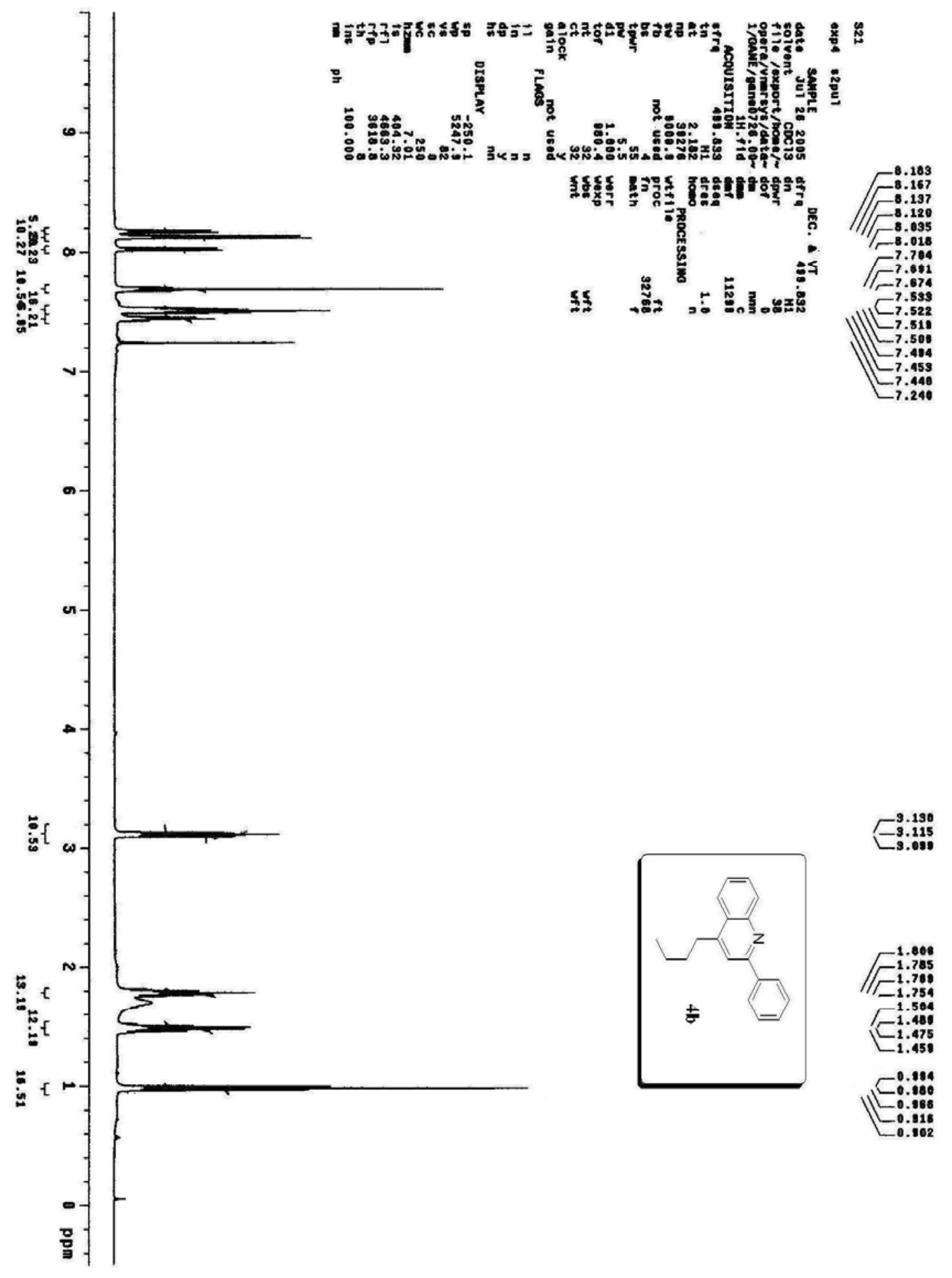




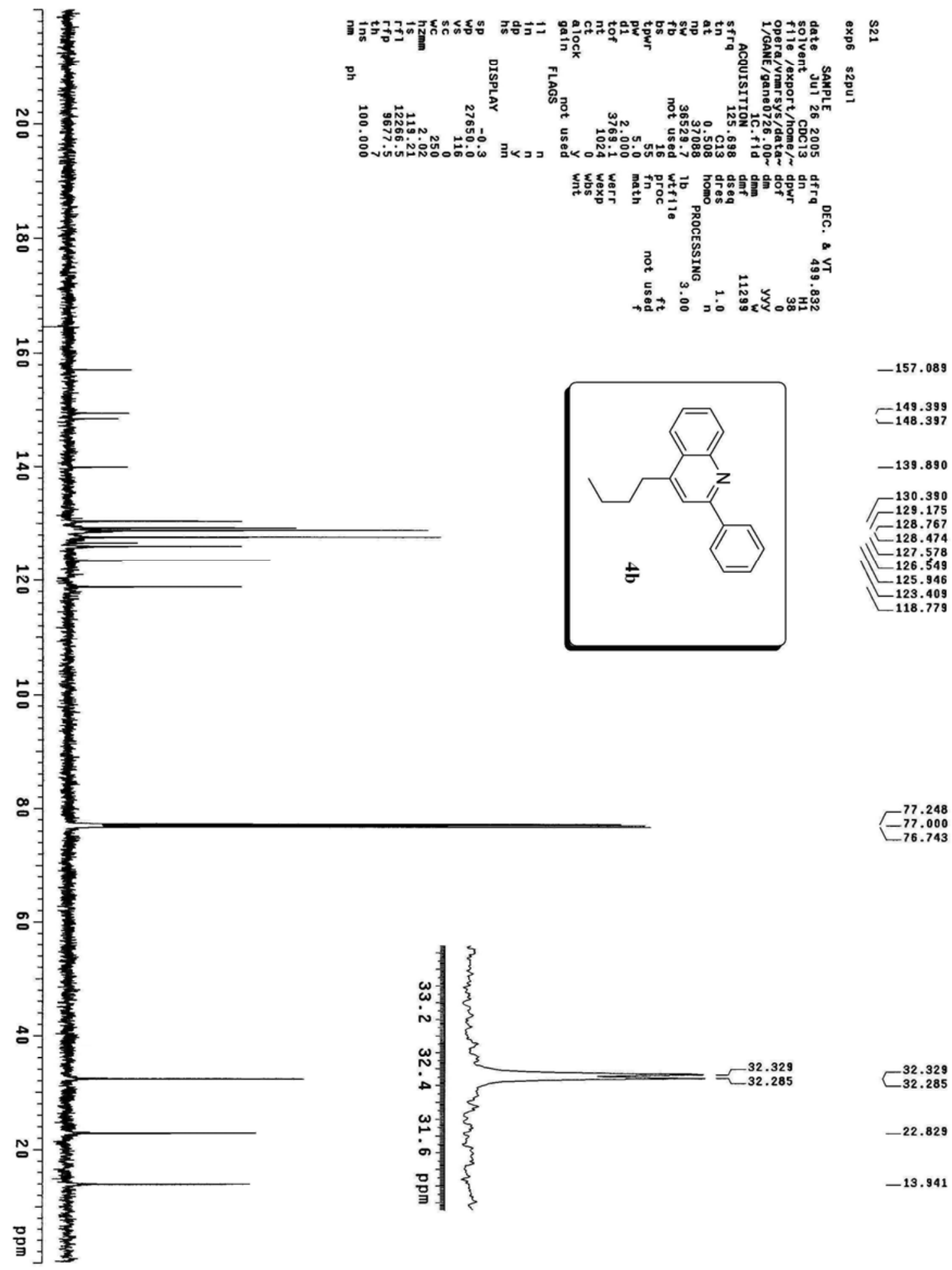



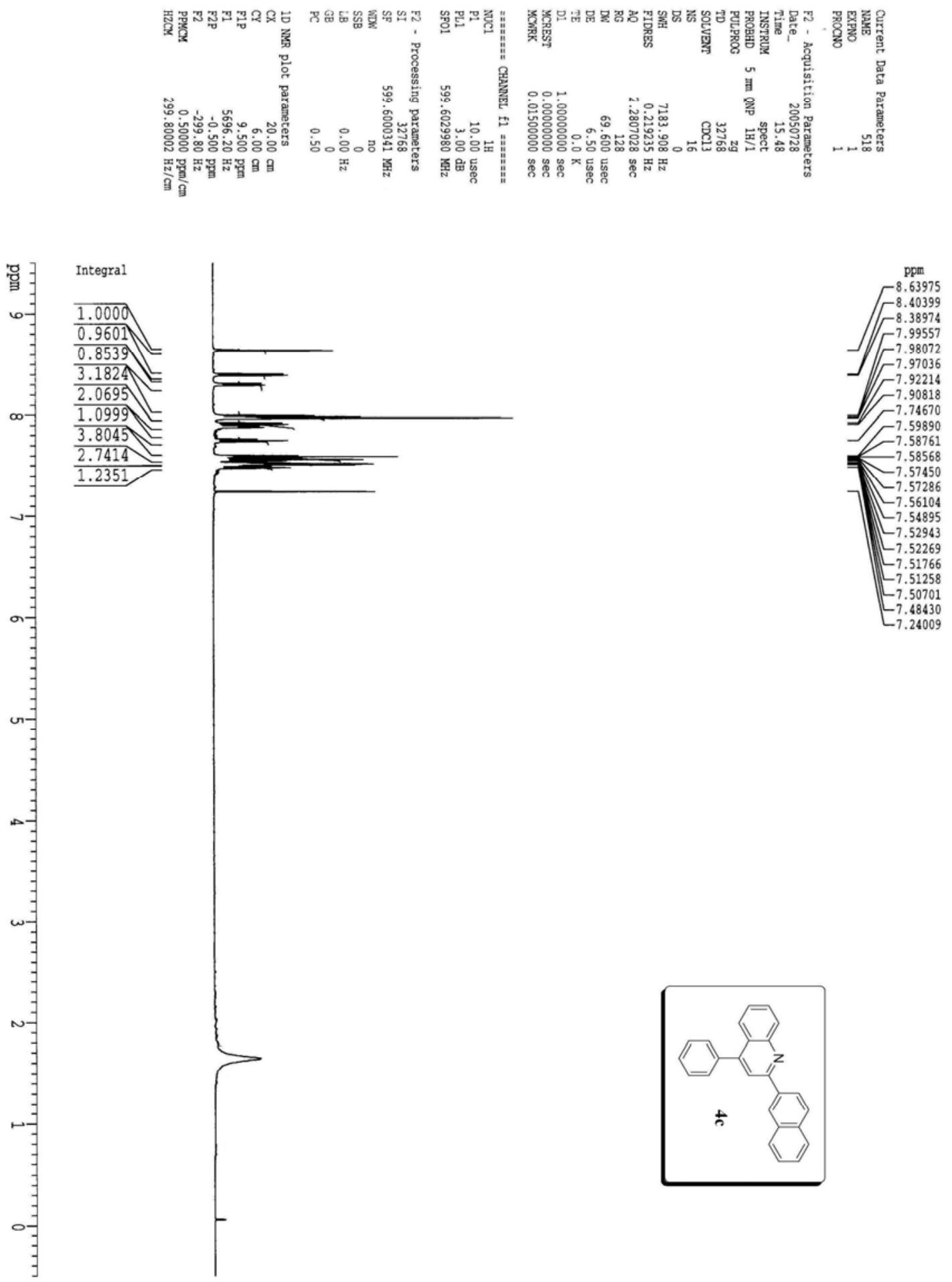

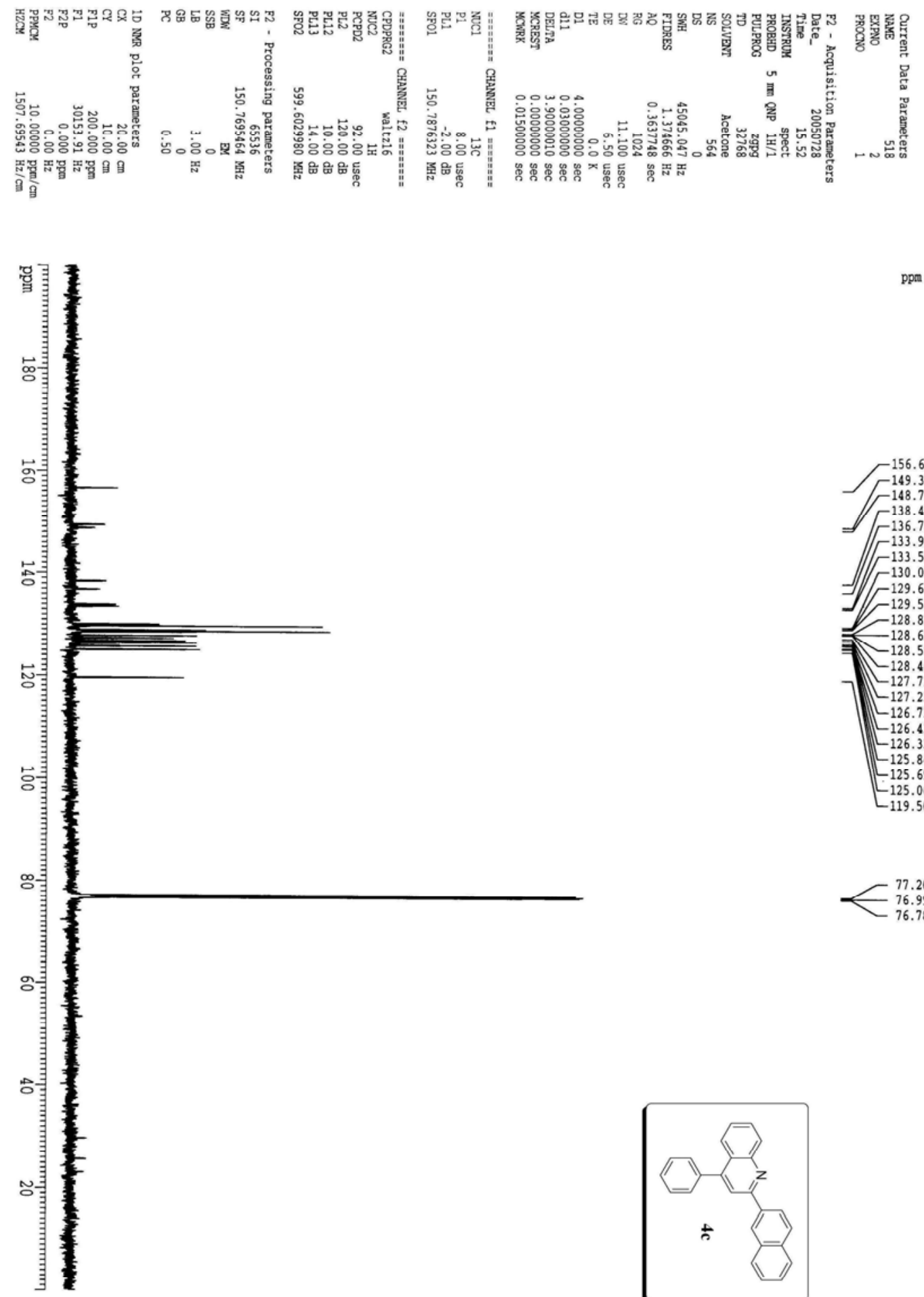

ppm
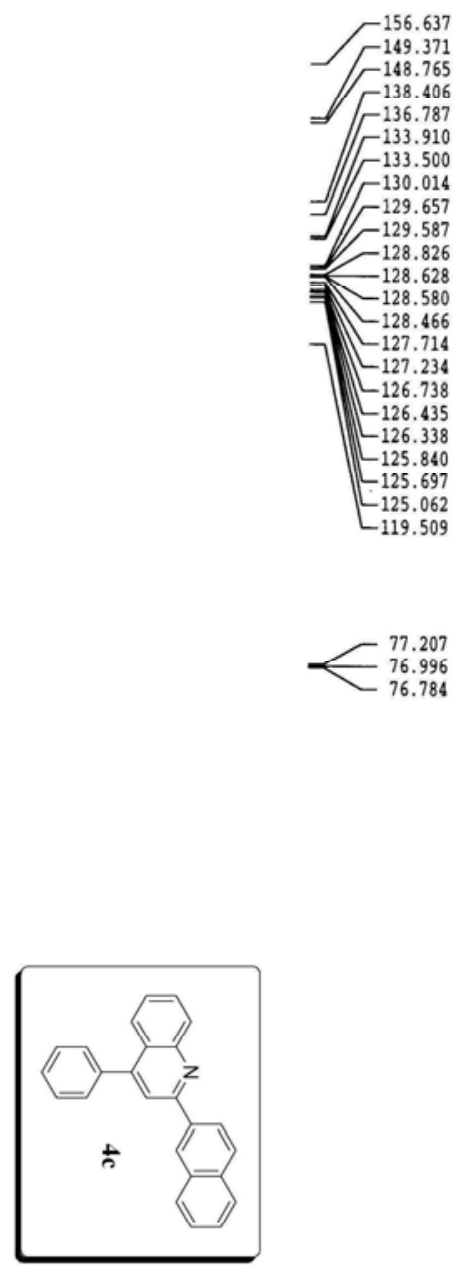

S-15 


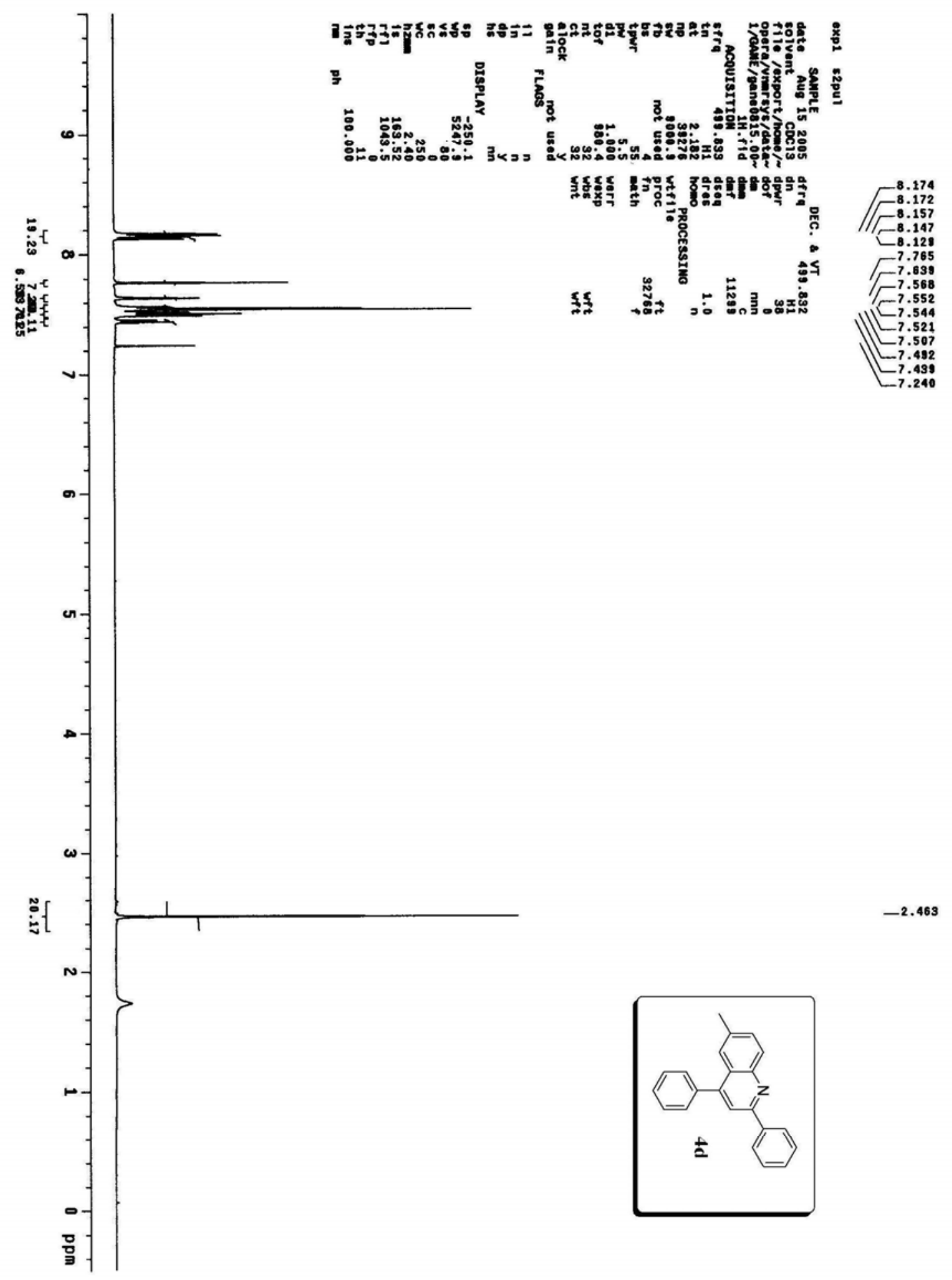

S-16 


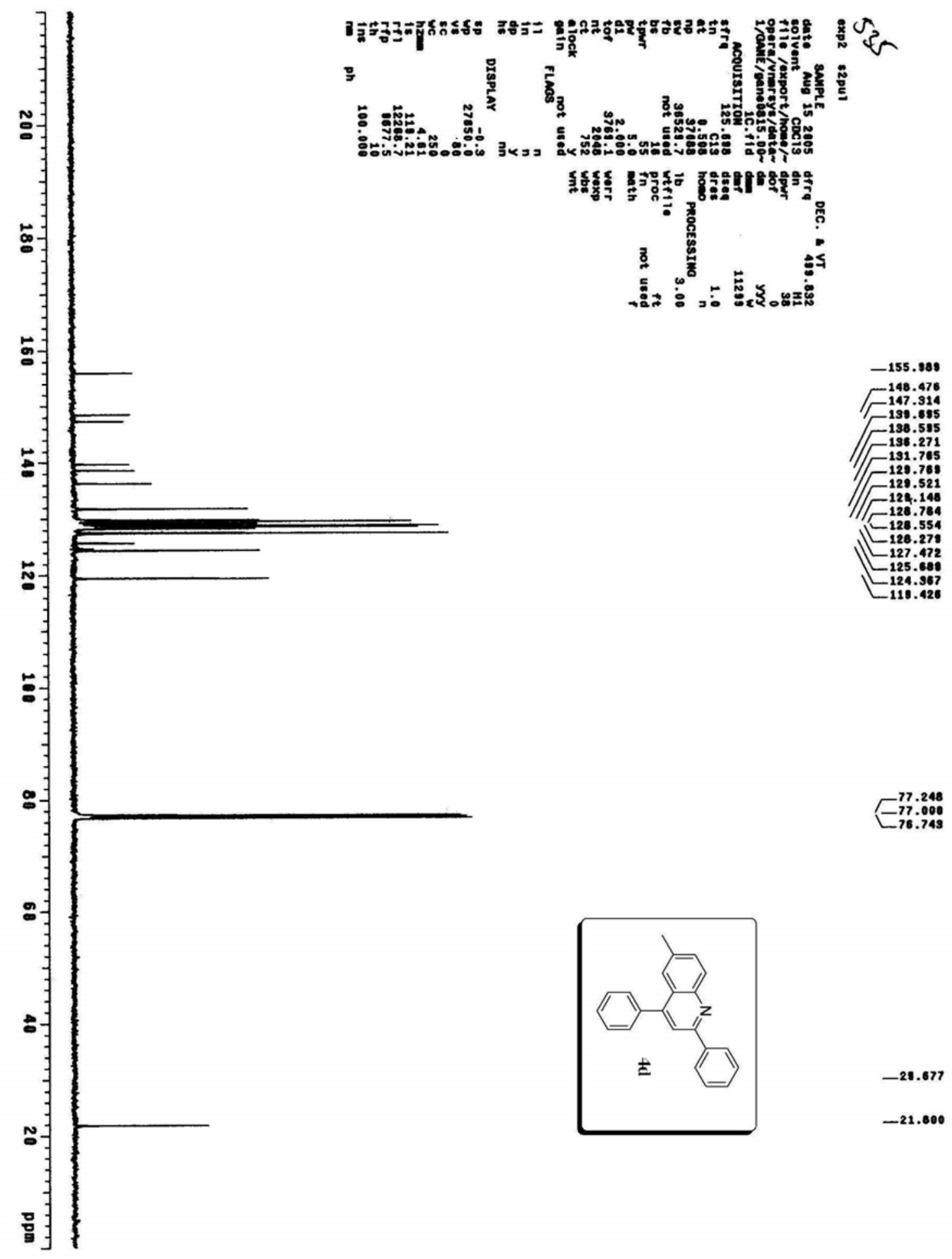




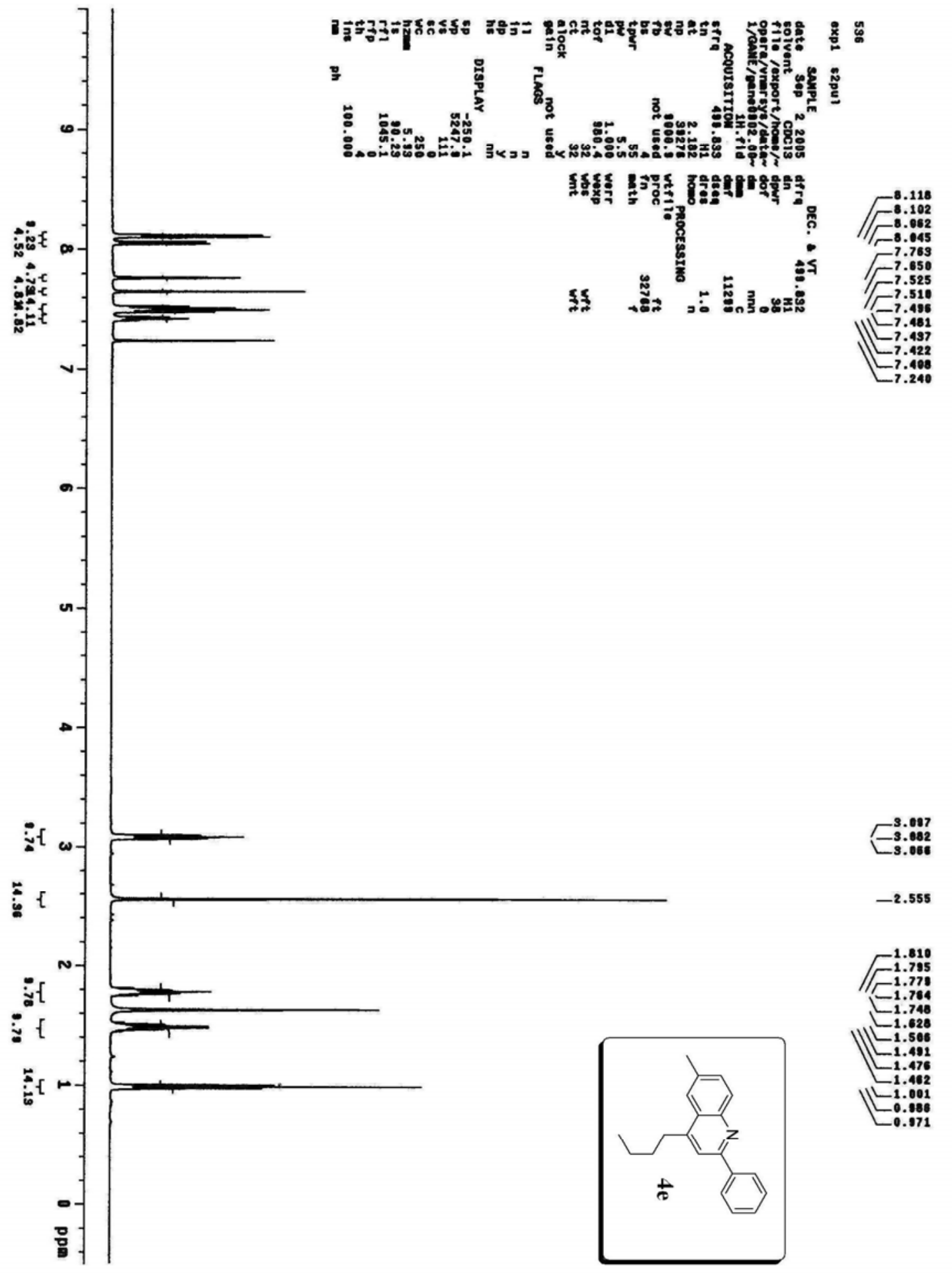




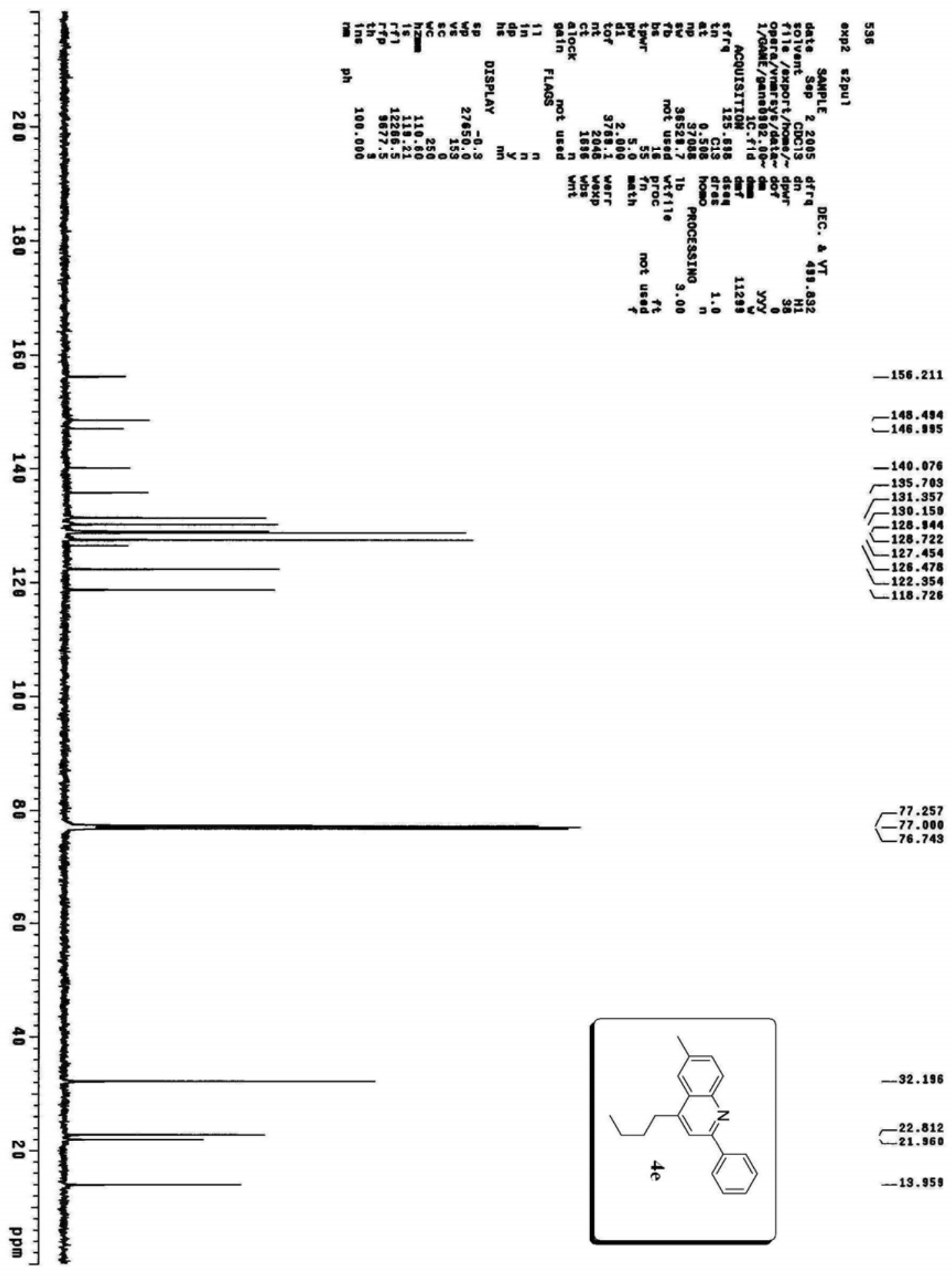



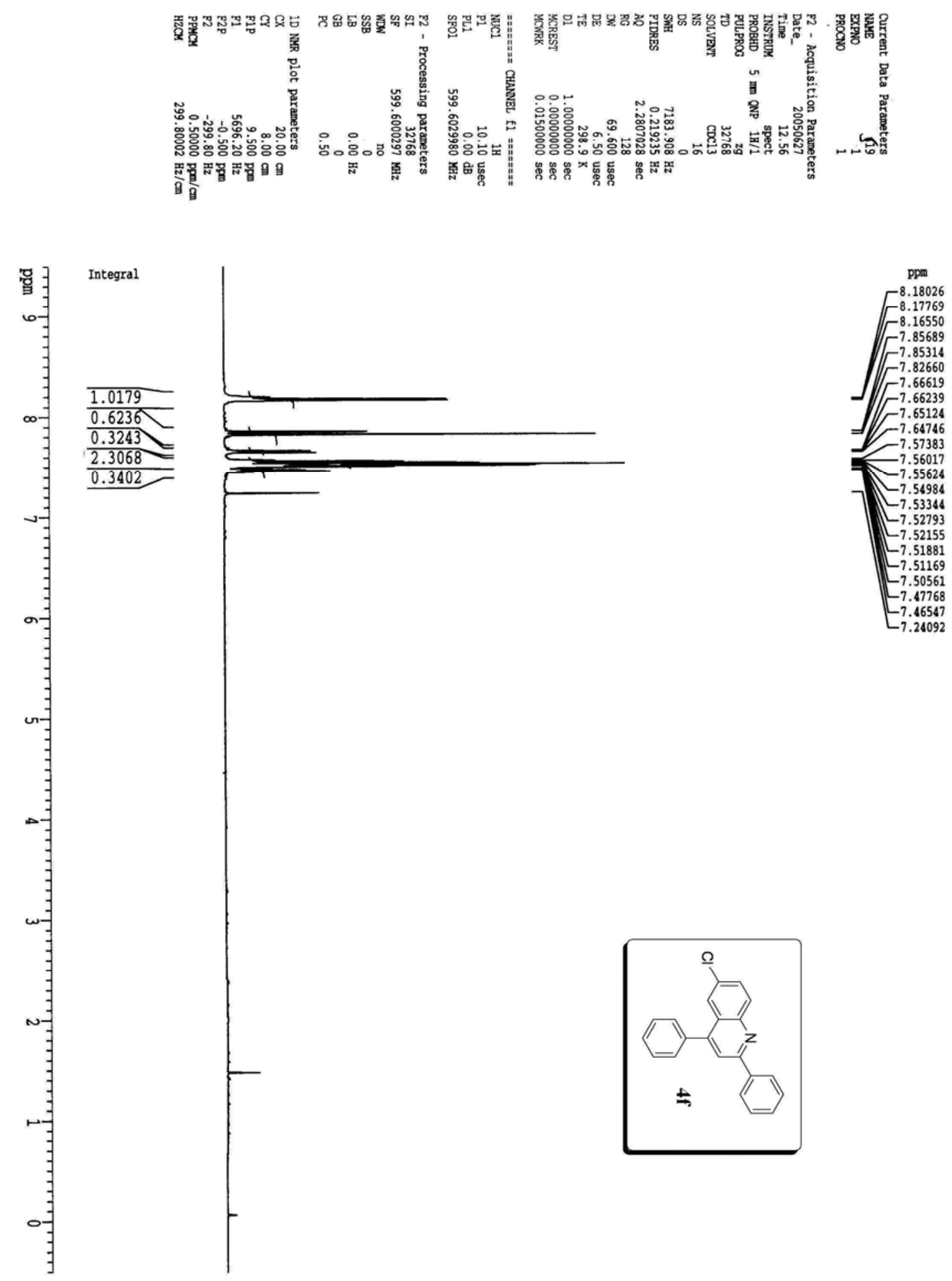

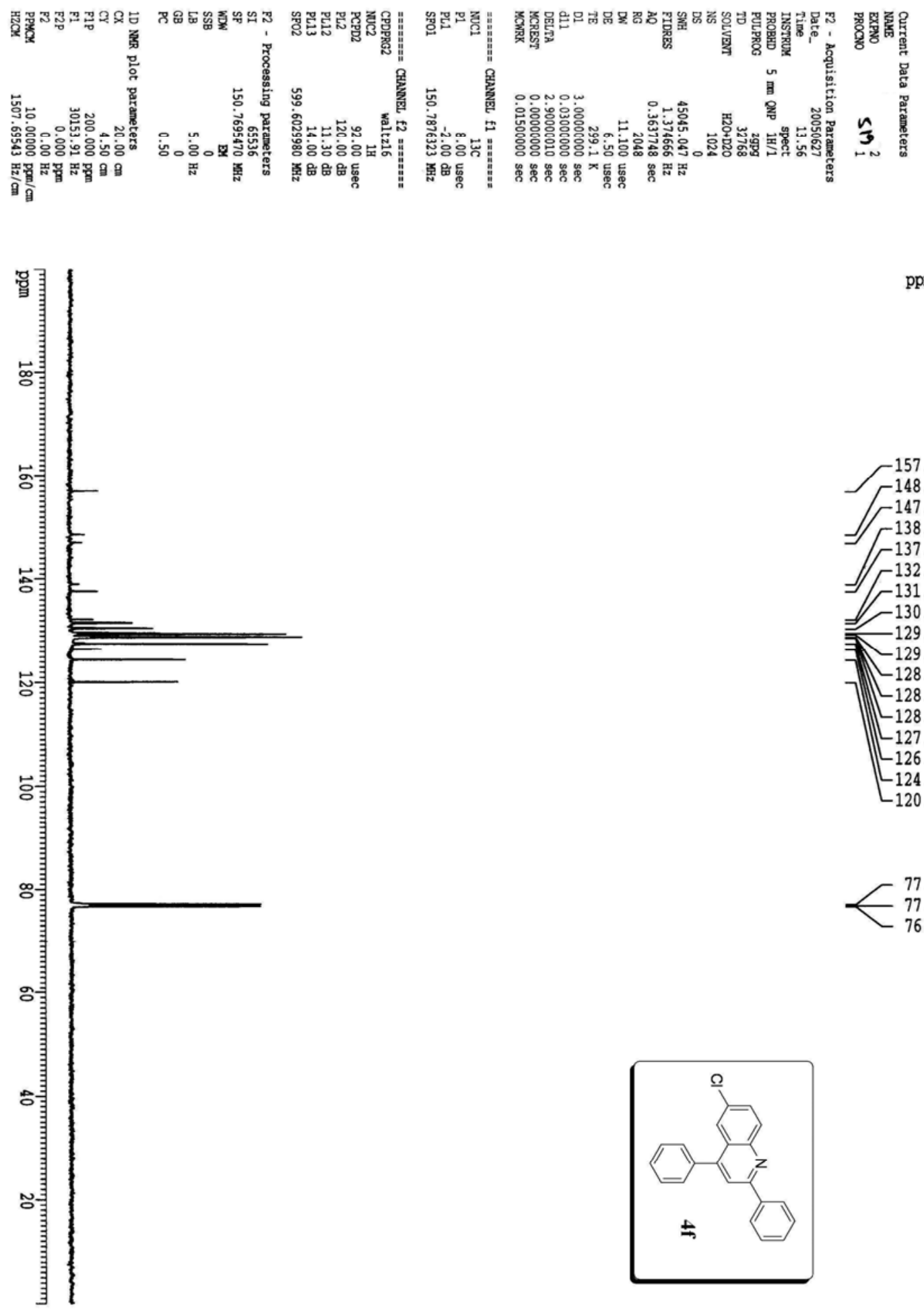

ppm
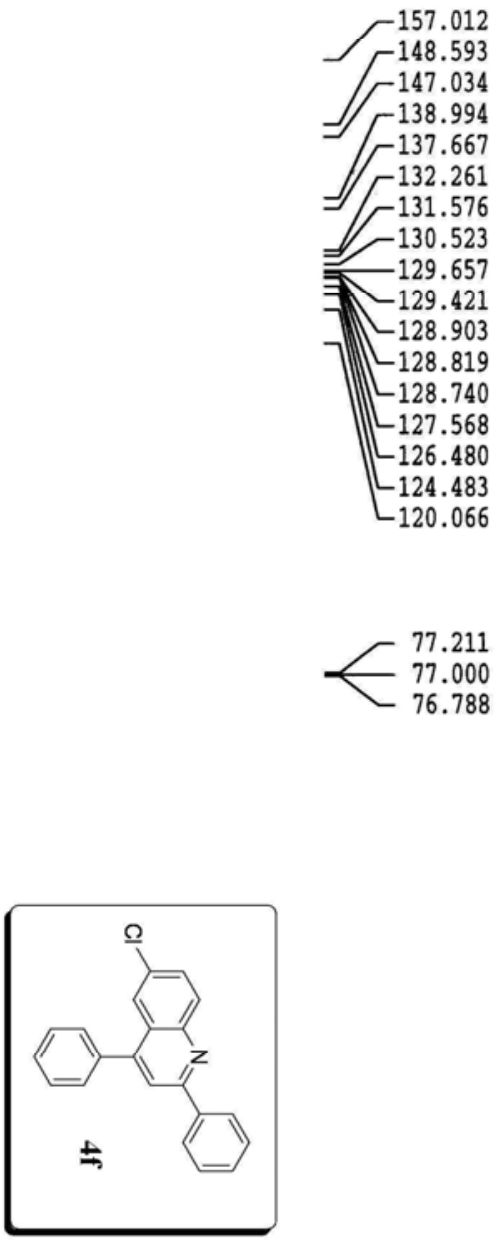


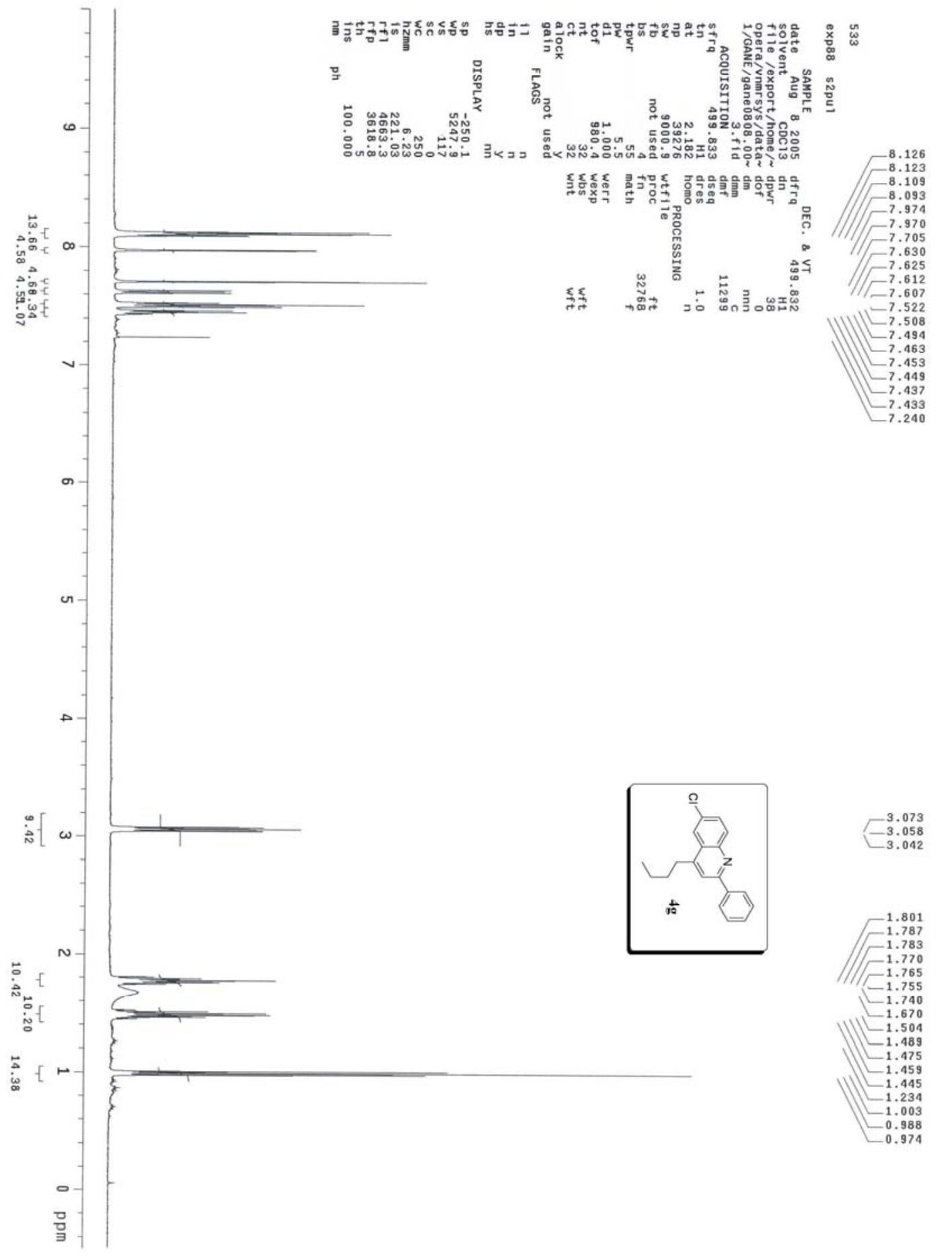




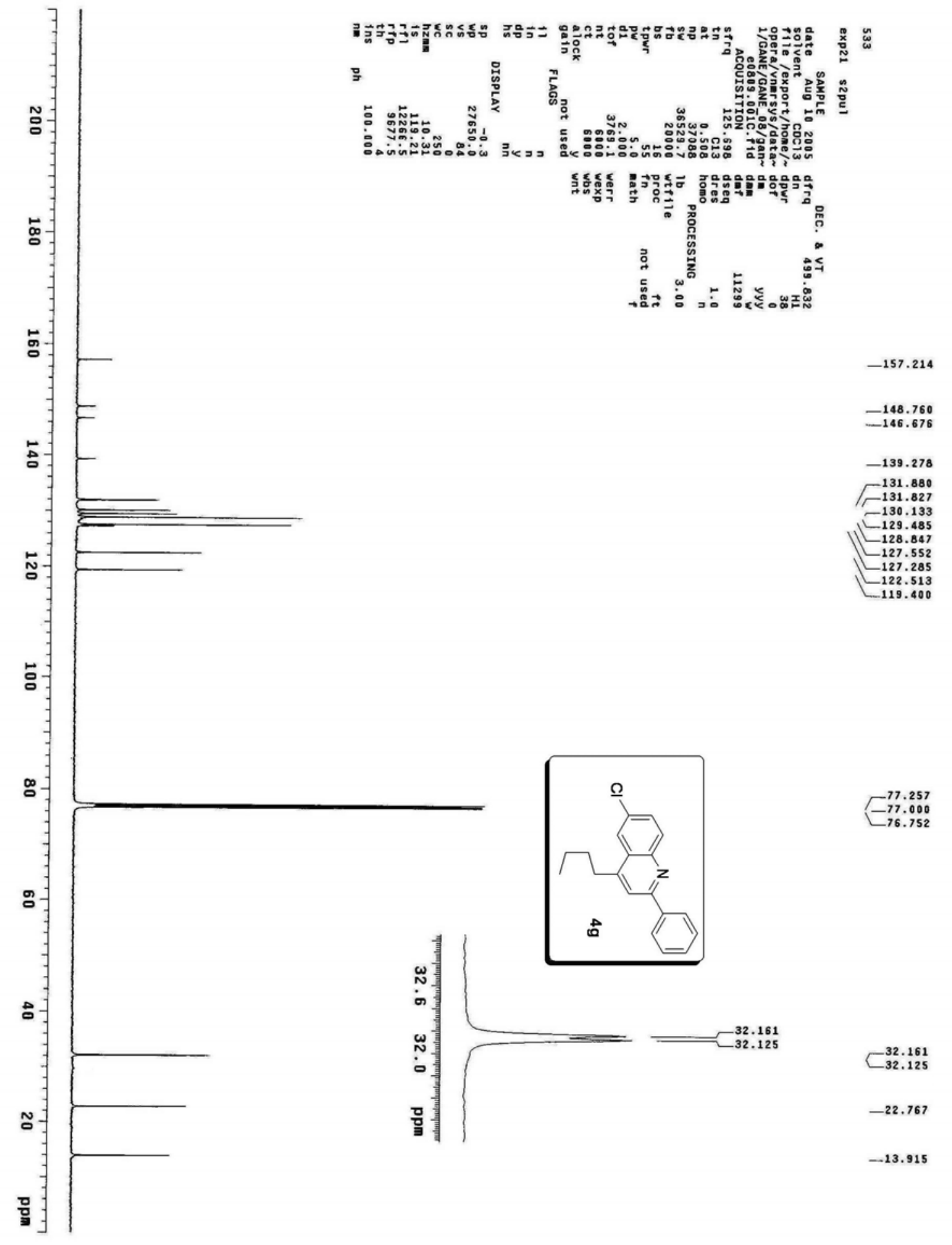




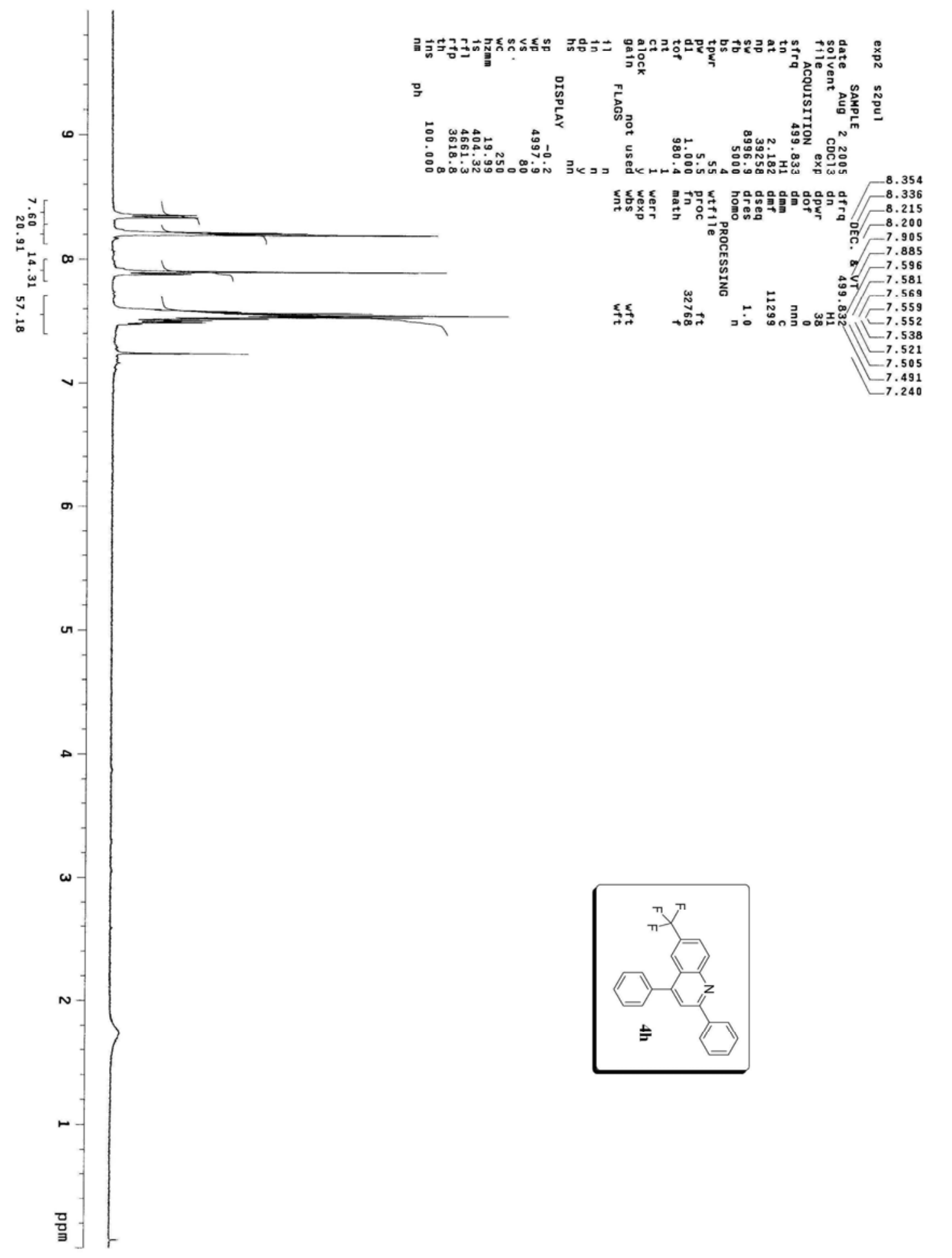




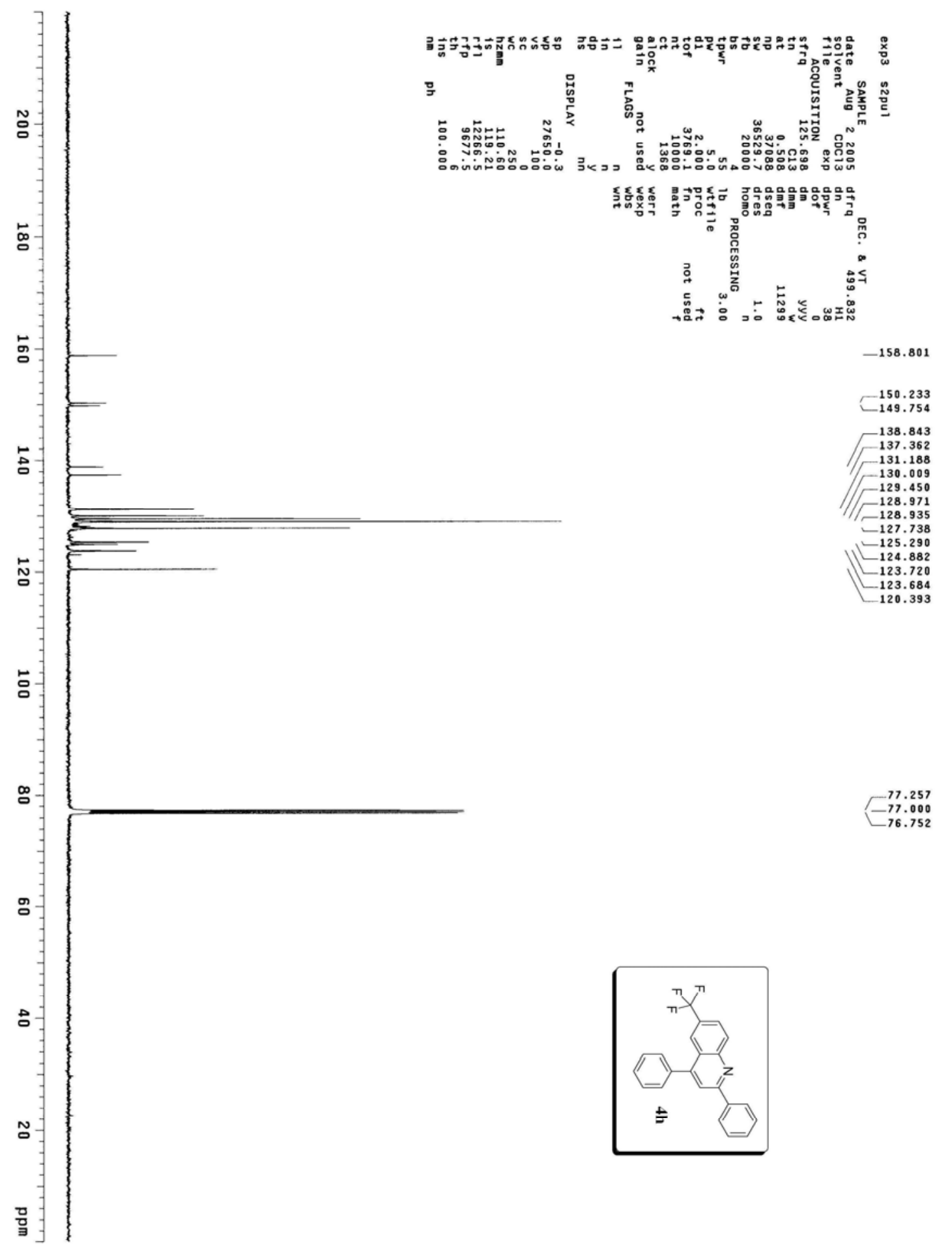



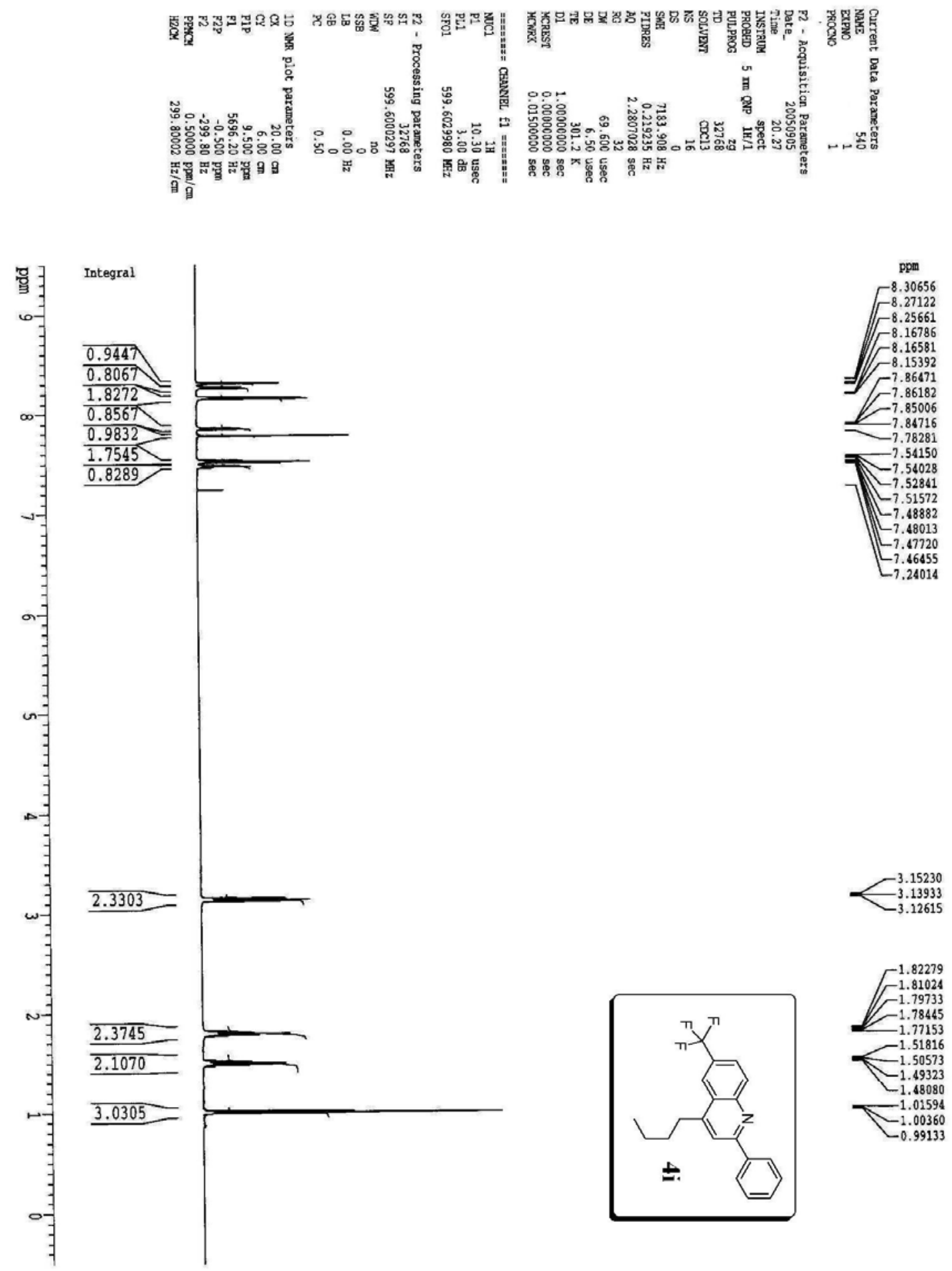


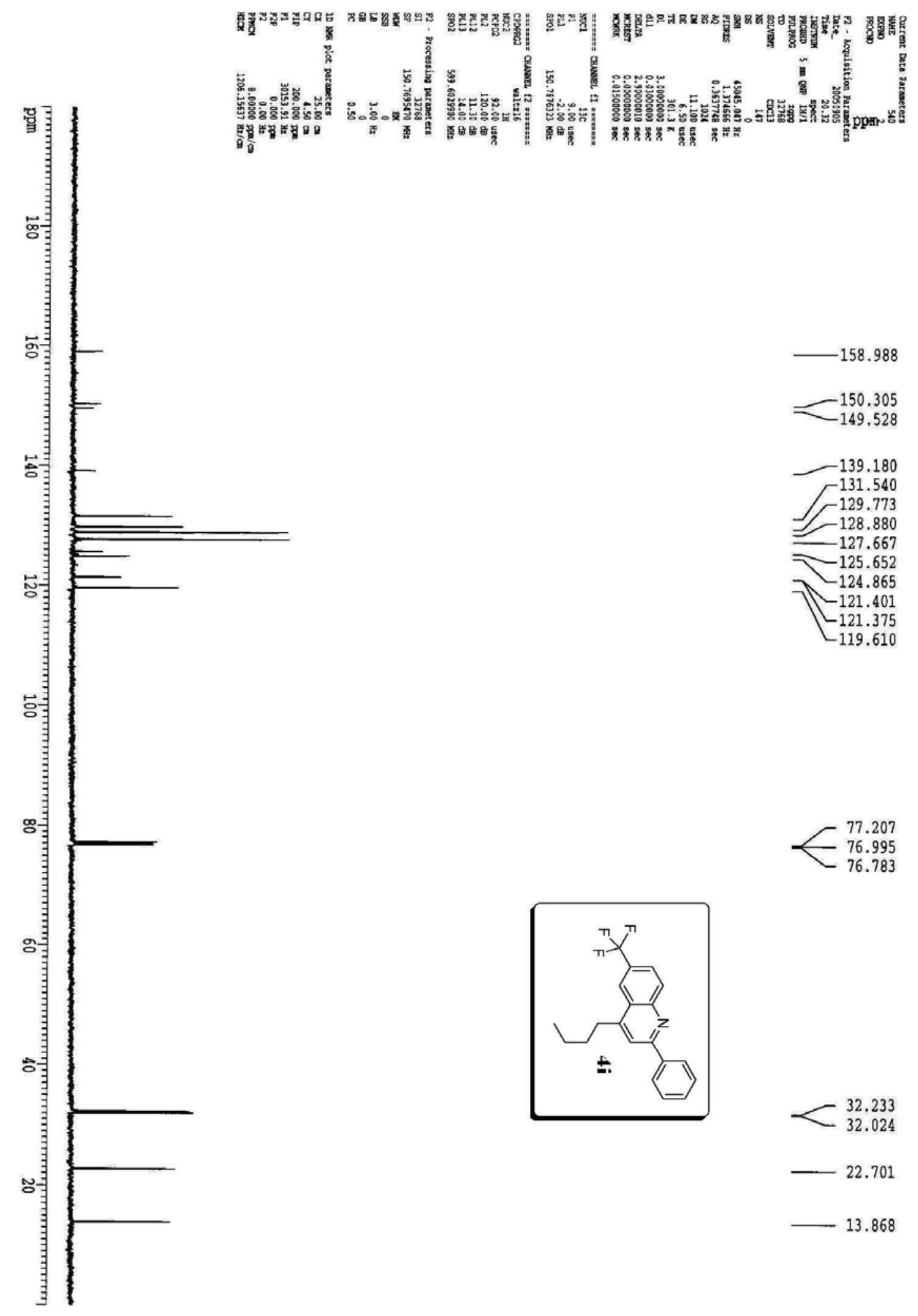




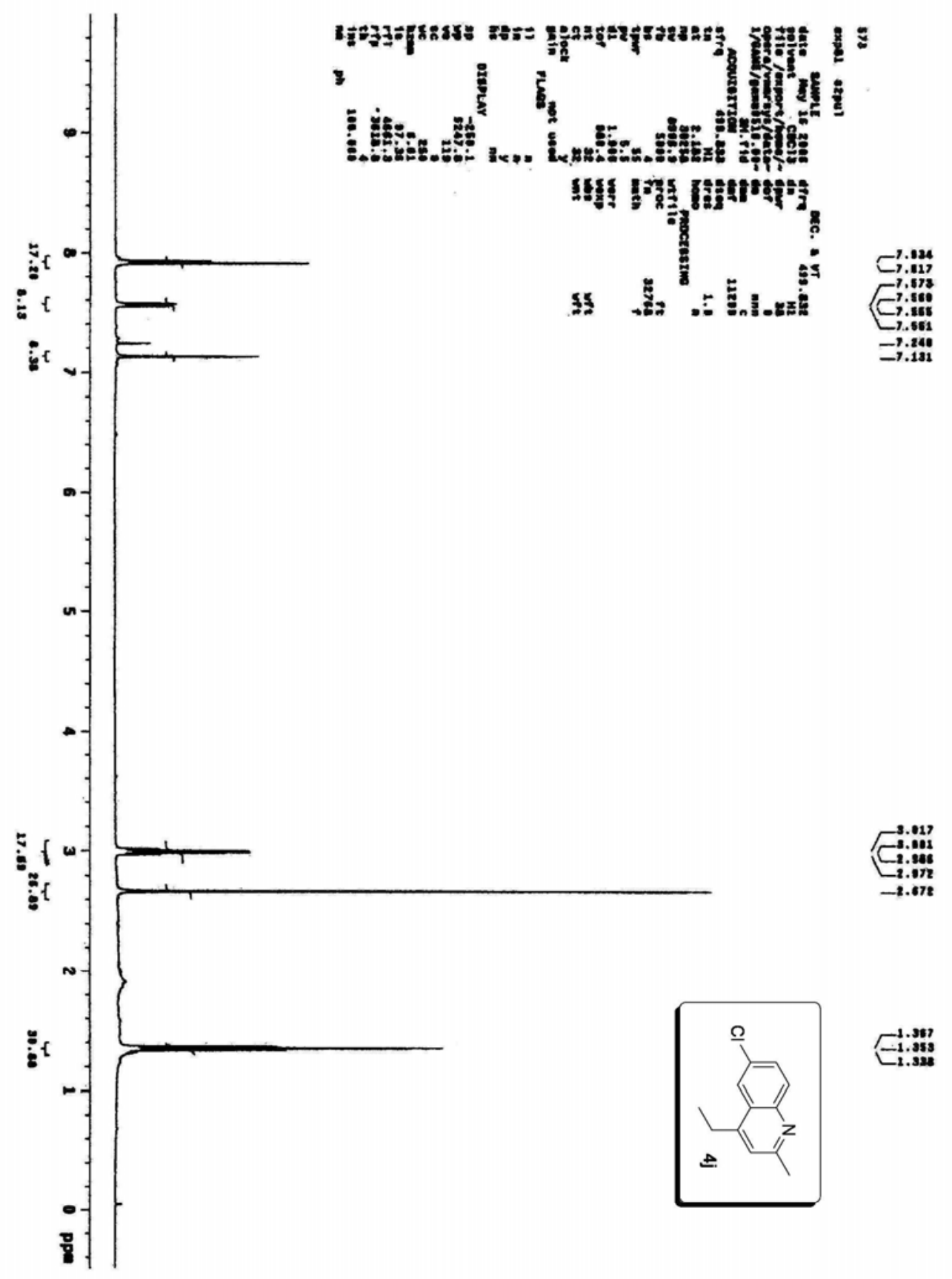




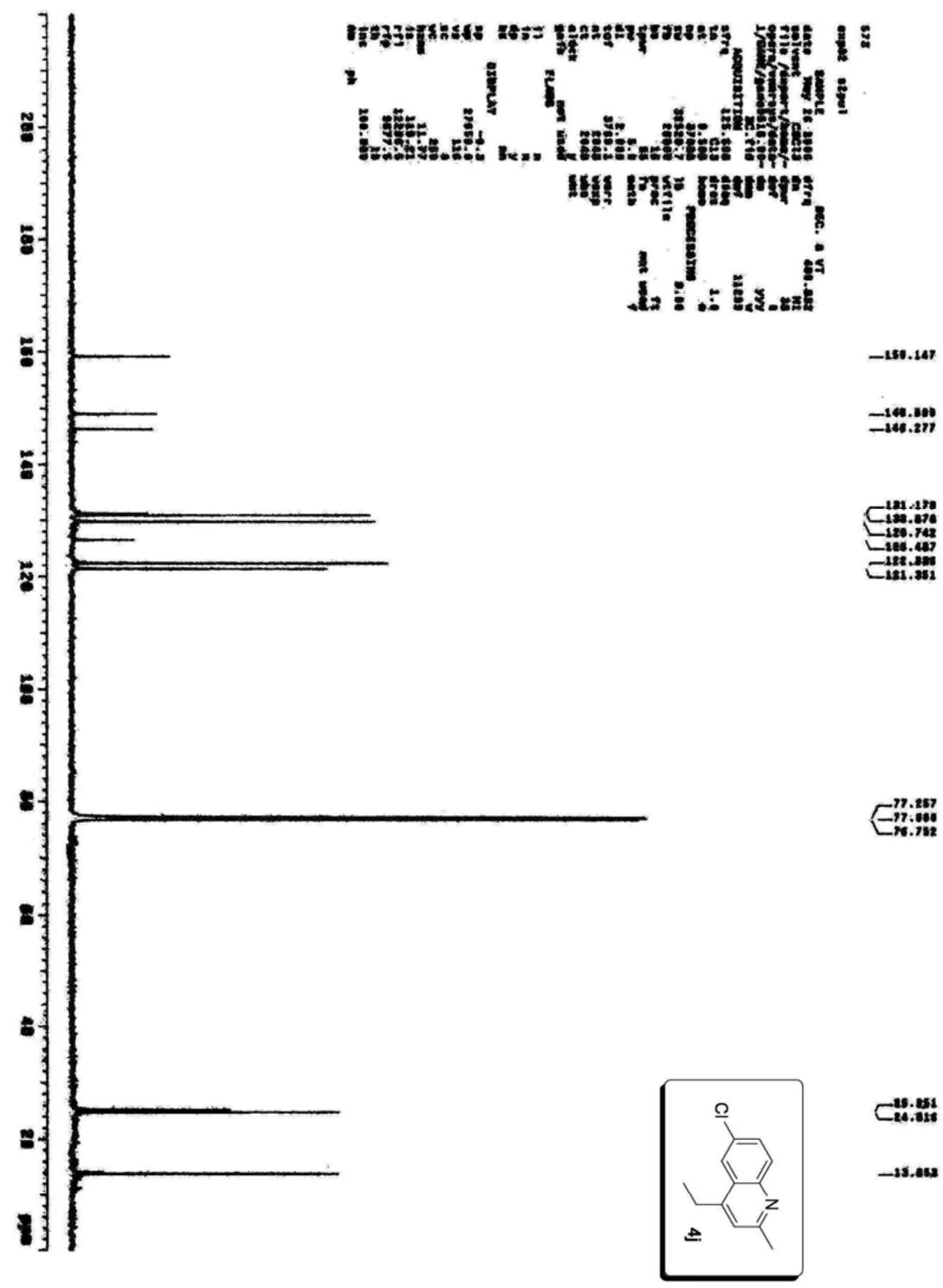




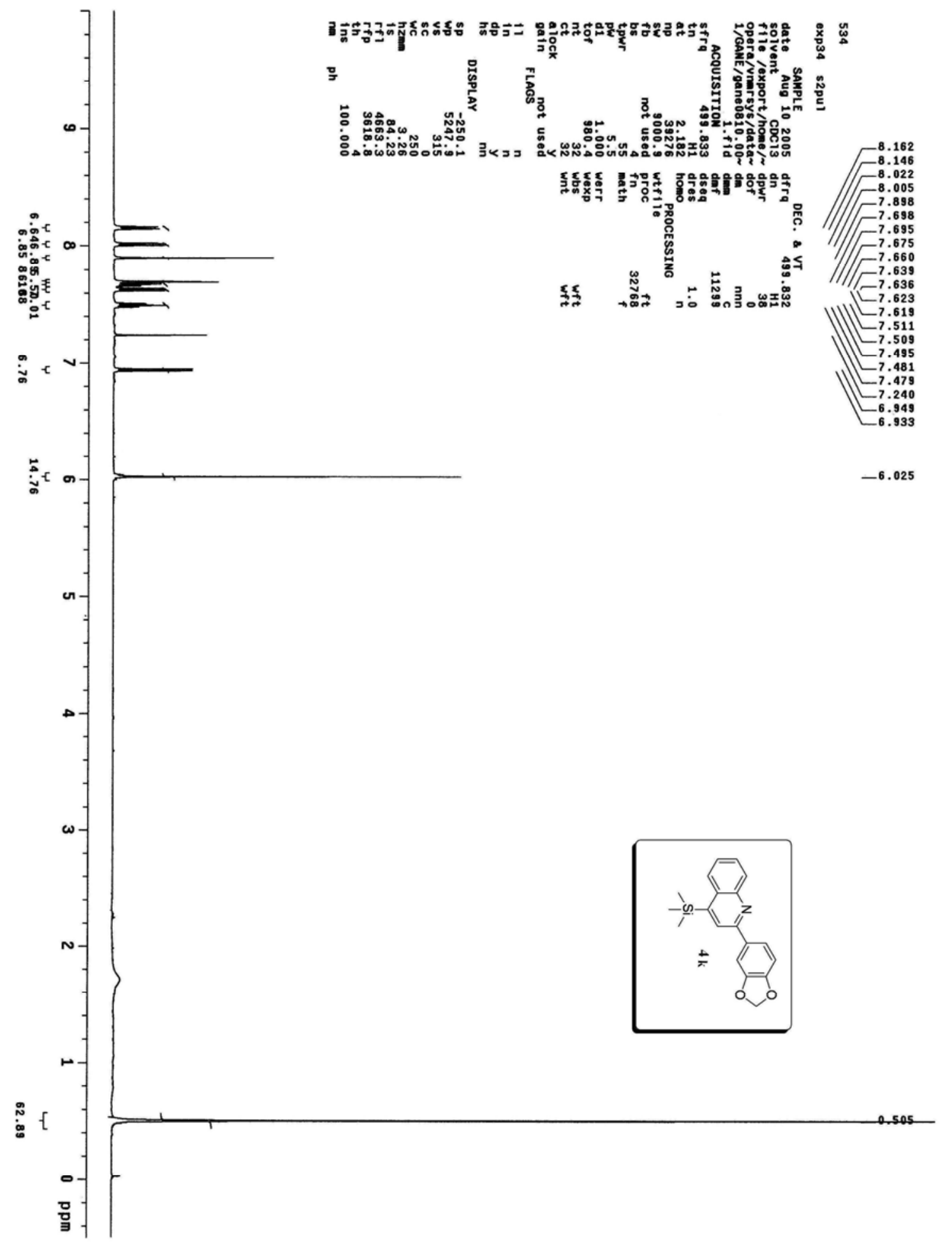




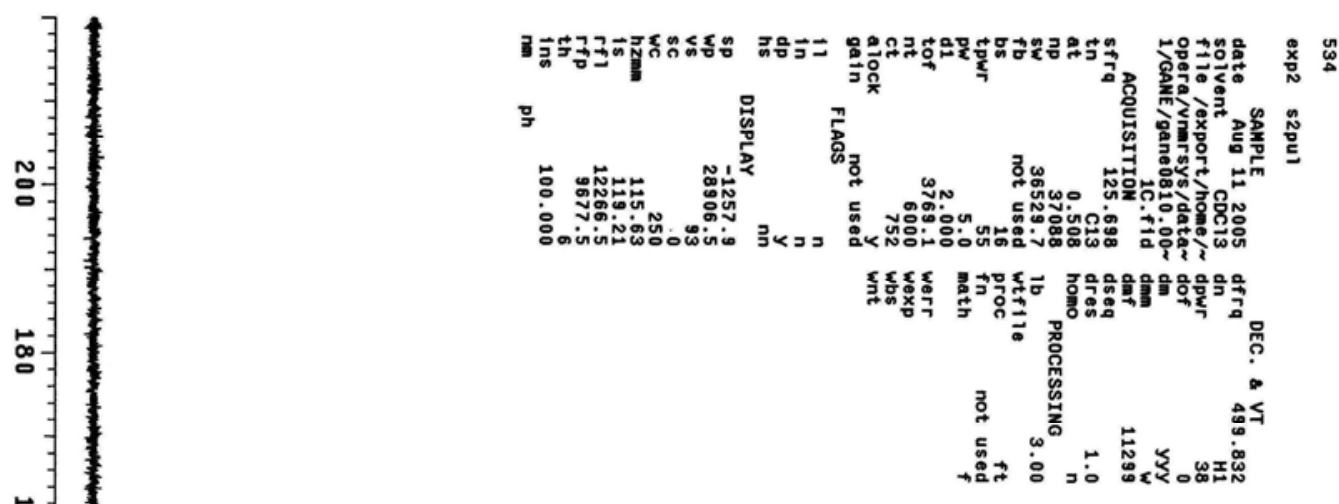

$-155.280$ 149.346
-148.743 148.334
-147.412

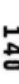

讨

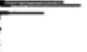

脑事 $\Gamma^{130.603}$ $\Gamma_{-130.399}^{139}$ -128.980
-127.578 $-127.578$ $-125.698$ -125.077
-121.839

$-108.489$ $-101.340$

ஃ

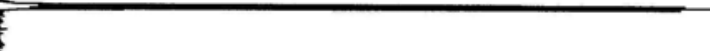

$\leftarrow_{76.743}^{77.257}$

淂

a-

客車

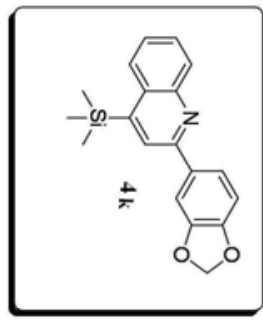

$--0.215$

뭌



I 


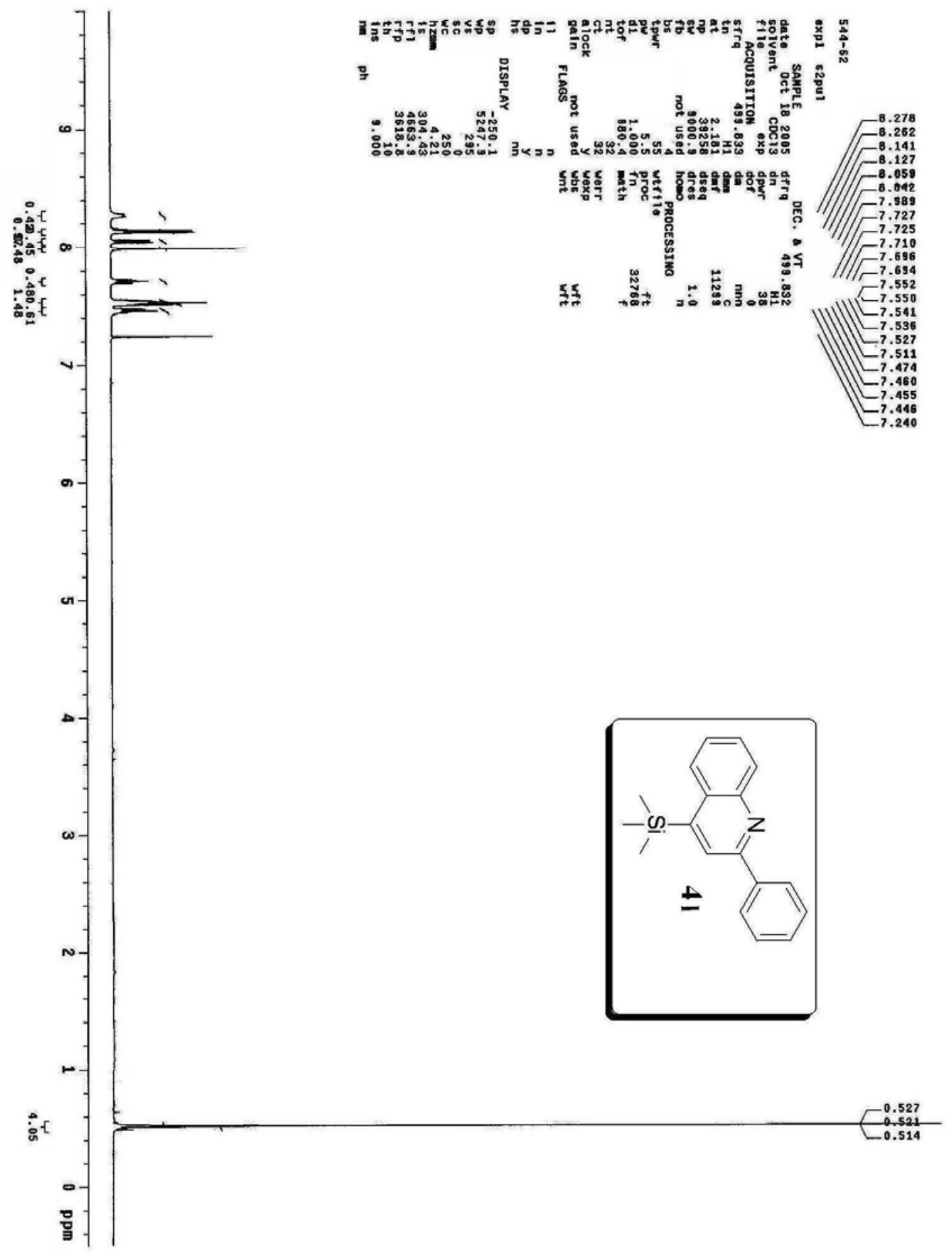




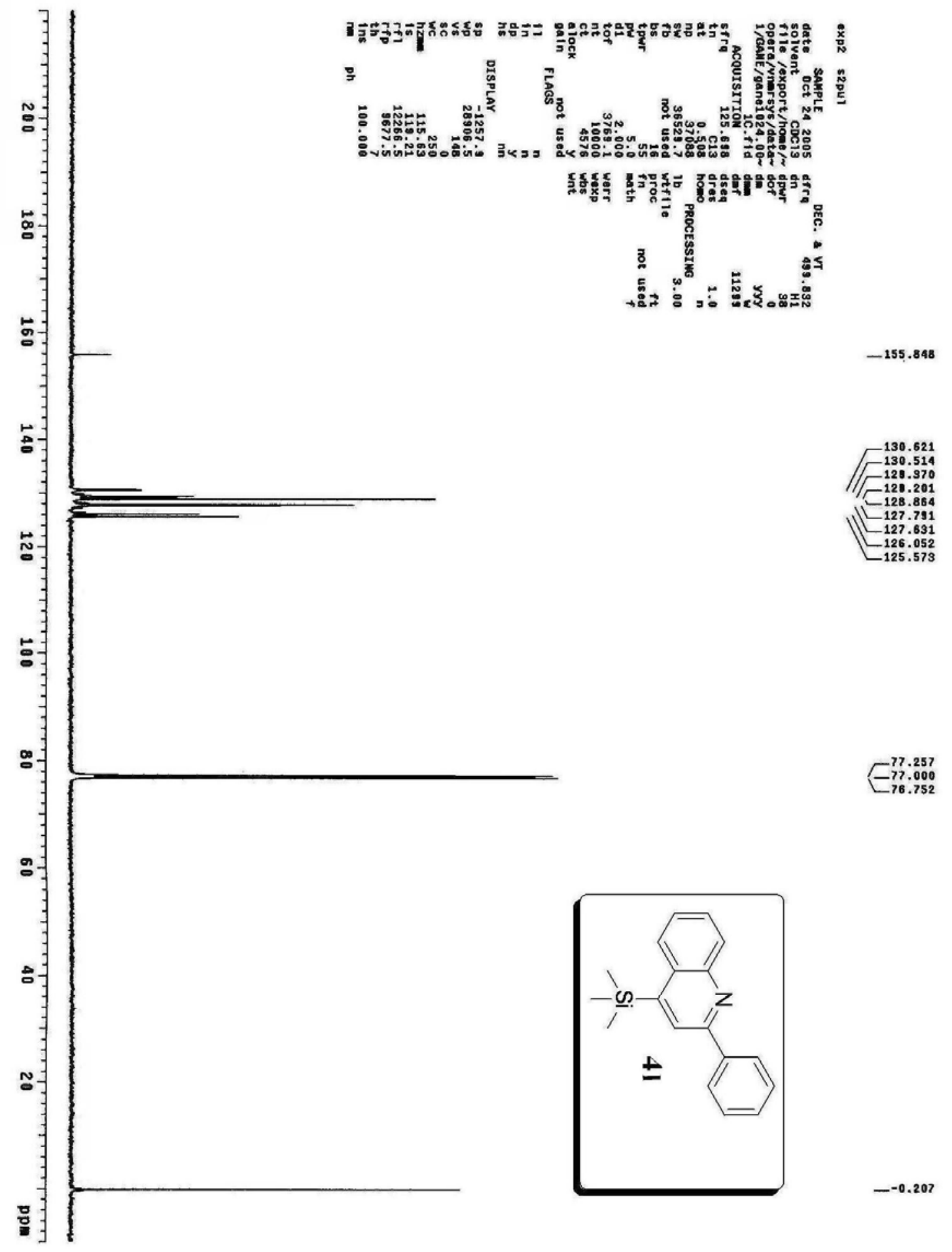




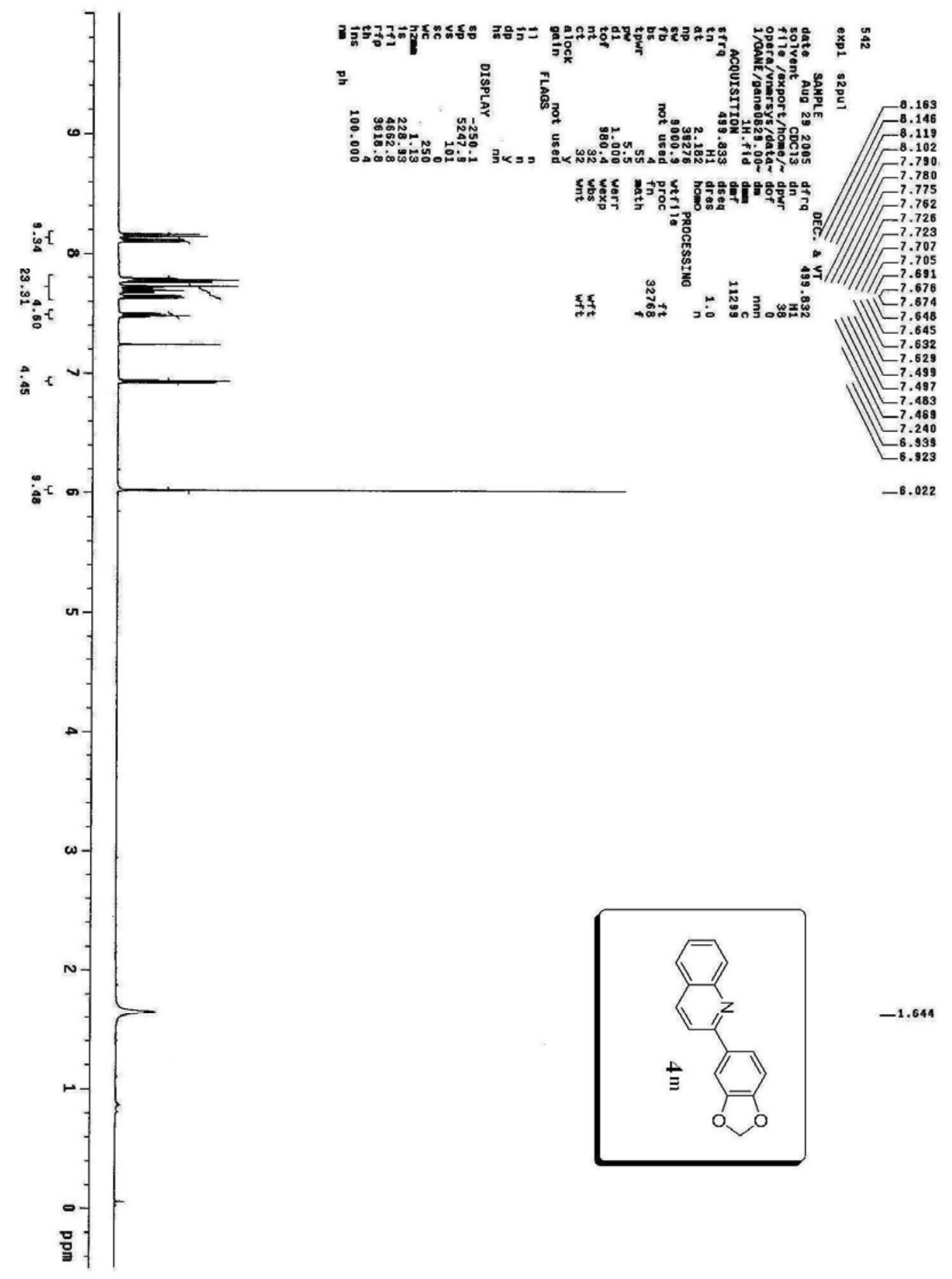




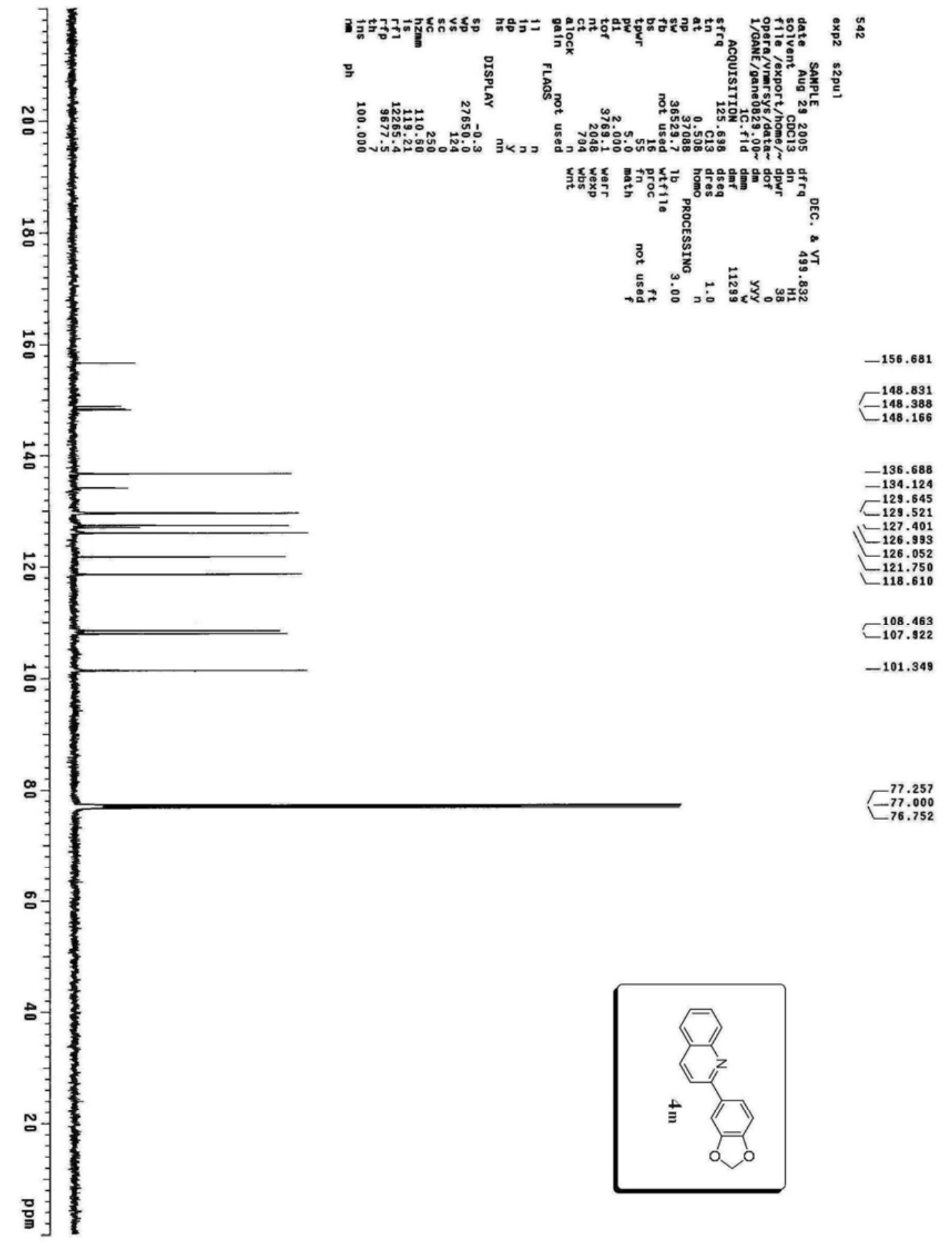




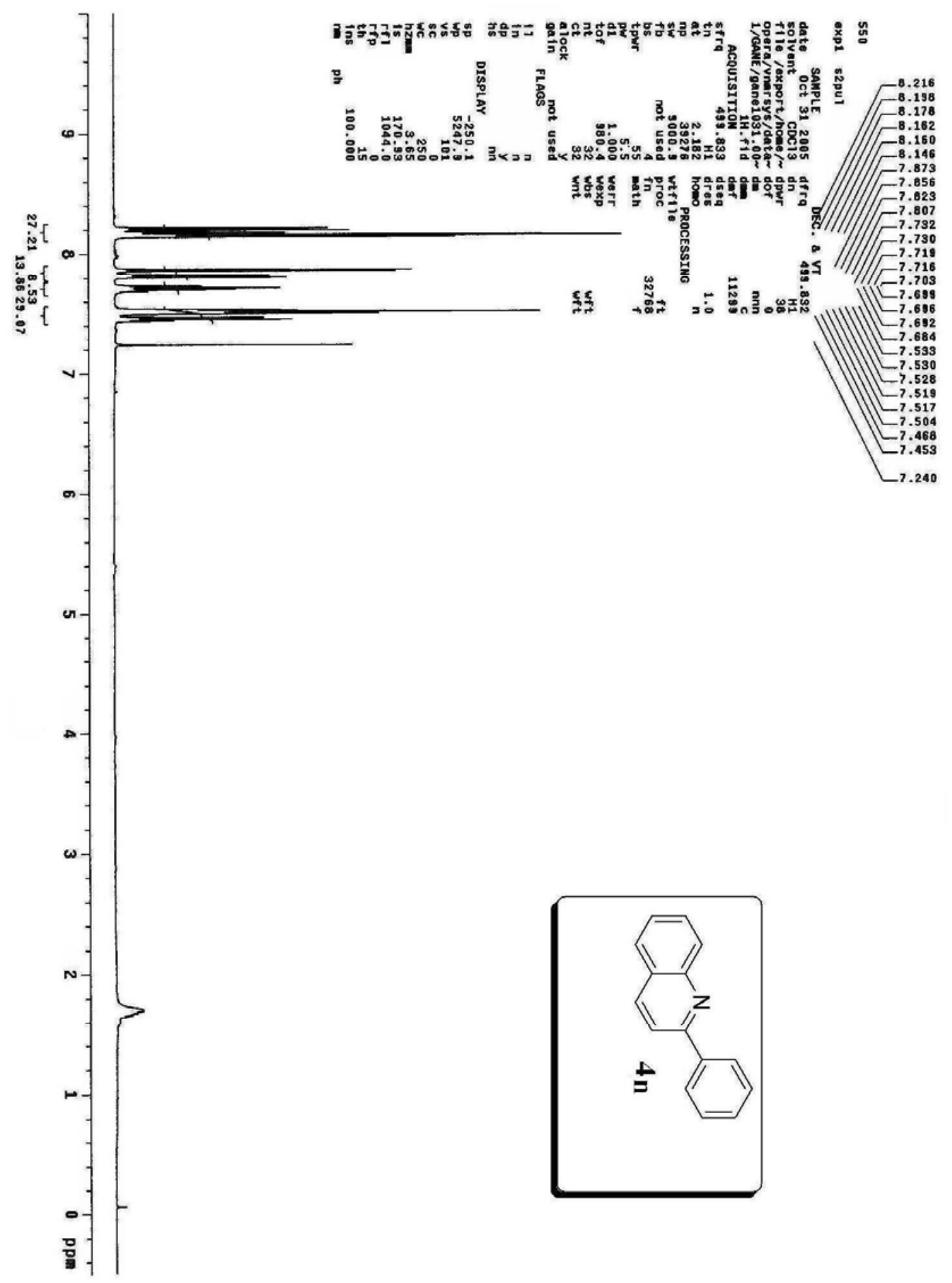




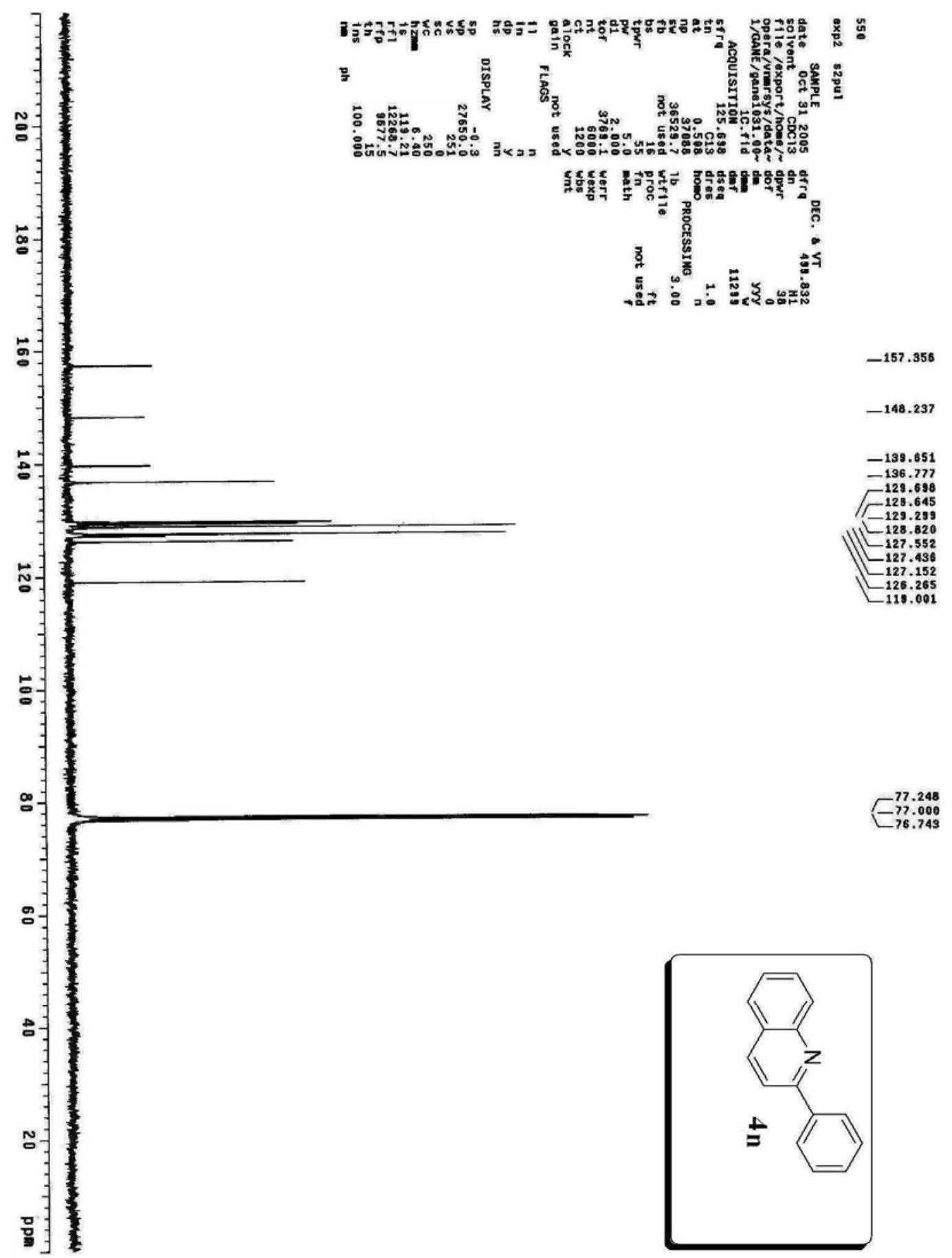

